FTUV-09-0226

$\operatorname{IPPP} / 09 / 13$

$\mathrm{DCPT} / 09 / 26$

CFTP/09-17

\title{
A combined beta-beam and electron capture neutrino experiment
}

\author{
José Bernabéu ${ }^{1}$, Catalina Espinoza ${ }^{1}$, \\ Christopher Orme $^{2}$, Sergio Palomares-Ruiz ${ }^{3}$ and Silvia Pascoli ${ }^{2}$ \\ 1 Universitat de València and IFIC, E-46100, València, Spain \\ 2 IPPP, Department of Physics, Durham University, \\ Durham, DH1 3LE, United Kingdom and \\ ${ }^{3}$ Centro de Física Teórica de Partículas, \\ Instituto Superior Técnico, 1049-001 Lisboa, Portugal
}

\begin{abstract}
The next generation of long baseline neutrino experiments will aim at determining the value of the unknown mixing angle, $\theta_{13}$, the type of neutrino mass hierarchy and the presence of CPviolation in the lepton sector. Beta-beams and electron capture experiments have been studied as viable candidates for long baseline experiments. They use a very clean electron neutrino beam from the $\beta$-decays or electron capture decays of boosted ions. In the present article we consider an hybrid setup which combines a beta-beam with an electron capture beam by using boosted Ytterbium ions. We study the sensitivity to the CP-violating phase $\delta$ and the $\theta_{13}$ angle, the CPdiscovery potential and the reach to determine the type of neutrino mass hierarchy for this type of long baseline experiment. The analysis is performed for different neutrino beam energies and baselines. Finally, we also discuss how the results would change if a better knowledge of some of the assumed parameters was achieved by the time this experiment could take place.
\end{abstract}




\section{INTRODUCTION}

In the past decade, atmospheric [1, 2], solar [3, 4, 5], reactor [6, 7, 8] and long-baseline accelerator [9, 10] neutrino experiments have provided compelling evidence for the phenomenon of neutrino oscillations. This has reshaped our understanding of the properties of elementary particles as it implies that neutrinos have mass and mix. The combined data can be described by two mass squared differences, $\Delta m_{31}^{2}$ and $\Delta m_{21}^{2}$, where $\Delta m_{j i}^{2}=m_{j}^{2}-m_{i}^{2}$, whose current best fit values are $\left|\Delta m_{31}^{2}\right|=2.4 \times 10^{-3} \mathrm{eV}^{2}$ and $\Delta m_{21}^{2}=7.65 \times 10^{-5} \mathrm{eV}^{2}[11]$. The two mixing angles $\theta_{12}$ and $\theta_{23}$ drive the solar and KamLAND, and atmospheric and MINOS neutrino oscillations, respectively, and are measured to be $\sin ^{2} \theta_{12}=0.304$ and $\sin ^{2} \theta_{23}=0.50$ [11]. The third mixing angle, $\theta_{13}$, is yet undetermined but is known to be small or zero. With available data, $\theta_{13}$ is constrained to be [11]

$$
\sin ^{2} \theta_{13}<0.040(0.056) \text { at } 2 \sigma(3 \sigma)
$$

It is interesting to note that very recently a first hint in favour of $\theta_{13} \neq 0$ has been found [12] in a combined analysis of atmospheric, solar and long-baseline reactor neutrino data, with:

$$
\sin ^{2} \theta_{13}=0.016 \pm 0.010 \quad \text { at } 1 \sigma
$$

implying a preference for $\theta_{13}>0$ at $90 \%$ CL. A different analysis [13] confirms the hint for $\theta_{13} \neq 0$ at $1.5 \sigma$ from the analysis of solar and KamLAND data owing to the latest SNO results, but not the one from the atmospheric data.

Although the experimental progress in neutrino physics over the last decade has been conspicuous, many of the fundamental questions surrounding neutrinos still need to be addressed. Understanding of the physics beyond the Standard Model responsible for neutrino masses and mixing requires knowledge of the nature of neutrinos (whether Dirac or Majorana particles), the neutrino mass ordering (normal or inverted), the absolute neutrino mass scale, the value of the unknown mixing angle $\theta_{13}$, and whether CP-symmetry is violated in the lepton sector. It will also be necessary to improve the precision on the known parameters, in particular to measure any deviation from maximal $\theta_{23}$ and, if so, to determine its octant.

Some of the issues above will be addressed by a future program of neutrino oscillation experiments [14, 15]. In particular, long baseline experiments using conventional beams [10] and nuclear reactors [16] will be the first to explore $\theta_{13}$ below the current limit and maybe 
confirm the hint for $\theta_{13} \neq 0[12]$. If $\theta_{13}$ is close to the present bound imposed by the running and near future experiments, the next generation of superbeams [17, 18], an extension of a conventional beam with an upgrade in intensity and detector size, and wide-band beams [19] will probe CP-violation and, for sufficiently long baseline, the neutrino mass hierarchy. For small values of $\theta_{13}$ or, if $\theta_{13}$ is large but a better precision on the neutrino parameters needs to be achieved, the community must turn to the novel concepts of the neutrino factory [20, 21] or beta-beam [22, 23]. Whereas conventional beams sourced from pion decays have an intrinsic contamination of electron neutrino at the $\sim 1 \%$ level (owing to kaons in the beam), neutrino factories and beta-beams will have clean sources from highly accelerated muons and ions, respectively, producing a well-collimated beam. In a neutrino factory, muons (antimuons) are produced, cooled and accelerated to a high boost before being stored in a decay ring. The subsequent decay sources a muon neutrino (muon antineutrino) and electron antineutrino (electron neutrino) which are aimed at magnetised detectors located a very long distance from the source. The use of magnetised detectors is necessary to separate the 'right muon' disappearance signal from the 'wrong muon' appearance signal, which is sensitive to matter effects and CP-violation. A beta-beam will exploit accelerated ions that $\beta$-decay sourcing a clean, collimated, electron neutrino beam. Magnetised detectors will not be necessary in this case, the only requirement being possession of good muon identification to detect the appearance channels. Therefore, water Čerenkov (WC), totally active scintillator, liquid argon detectors and non-magnetised iron calorimeters could be used, depending on the peak energy.

The determination of the oscillation parameters is severely affected by degeneracies [24, 25, 26, 27, 28]; the possibility that different sets of the unknown parameters $\left(\operatorname{sgn}\left(\Delta m_{31}^{2}\right), \delta, \theta_{13}, \theta_{23}\right.$ octant) can provide an equally good fit to the probability for neutrino and antineutrino oscillations, for fixed baselines and energy. Therefore, a high precision measurement of the appearance probabilities is not sufficient to discriminate the various allowed solutions. In order to weaken or resolve this issue, various strategies have been put forward: exploiting the energy dependence of the signal in the same experiment [19, 29], using reactor neutrino experiments with an intermediate baseline [30], combining different long baseline experiments [31], adding the information on $\theta_{13}$ from reactor experiments [32], or using more than one baseline for the same beam [33, 34, 35, 36, 37, 38]. In addition, $\theta_{13}$ controls the Earth matter effects in multi-GeV atmospheric [39, 40, 41, 42, 43] and in supernova neutrino 
oscillations [44] (see also Ref. [45]). These might provide useful information on the type of neutrino mass hierarchy and $\theta_{13}$; the magnitude of the T-violating and CP-violating terms in neutrino oscillation probabilities is directly proportional to $\sin \theta_{13}$ [46, 47].

In beta-beam experiments, the energy dependence of the signal is typically used to extract information on the mass hierarchy and CP-violation. Matter effects increase with baseline and energy suggesting that setups with baselines $>600 \mathrm{~km}$ are necessary [29, 38, 48, 49, 50, 51, 52] for the determination of the type of neutrino mass ordering. Such strategies would make use of a proposed upgrade to the CERN Super Proton Synchrotron (SPS) which would equip the accelerator with fast superconducting magnets allowing high boosts and fast ramps. The latter are important to reduce the loss of ions through decay in the acceleration stage. A sister approach to the beta-beam is to use the neutrinos sourced from ions that decay mainly through electron capture [53, 54, 55, 56]. If the electron capture decay is dominated by a single channel, then a monoenergetic electron neutrino beam can be produced this way. In this case, all the beam intensity can be concentrated at the appropriate energy to get the best sensitivity to the oscillation parameters. In order to disentangle the CP violating phase with neutrino beams only, one makes use of the different energy dependence of the CP-even and CP-odd terms in the appearance probability [47]. Electron capture competes with $\beta^{+}$-decay when the $Q_{\mathrm{EC}}$-value $>2 m_{e}, m_{e}$ being the electron mass. With the ions identified in [53], the use of an upgraded SPS or the Tevatron ${ }^{1}$ is necessary to source baselines in excess of CERN-Frejus $(130 \mathrm{~km})$. In this paper, we discuss a hybrid of these two approaches. ${ }^{2}$ By selecting a nuclide with $Q_{\mathrm{EC}} \sim 4 \mathrm{MeV}$, we can make use of neutrinos from an electron capture $\delta$-spike and $\beta^{+}$continuous spectrum simultaneously. Assuming a detector with low energy threshold, the use of such ions allows one to exploit the information from the first and second oscillation maxima with a single beam, in a similar way to the approach used in Ref. [29]. There the spectral information was used to remove some of the degeneracies and reach physics sensitivities comparable to the scenarios with a neutrino and antineutrino beam that are often presented in the literature. The use of the hybrid

\footnotetext{
${ }^{1}$ Note that the present Tevatron configuration does not ramp fast enough, resulting in a high loss of ions, so this might not be a very realistic experimental setup, at least in the present configuration.

${ }^{2}$ Note that the use of this hybrid approach was first mentioned in Ref. [55], although the proposal, unlike the present case, was to use long-lived ions. In addition, the phenomenology of this approach was not studied.
} 
approach we propose makes it possible to use a monochromatic beam at higher energies and a beta-beam at lower energies. The need for good neutrino energy resolution at the higher energies will therefore be less crucial than for high- $\gamma$ beta-beam scenarios.

The paper is organised as follows. In Sec. II, we introduce the hybrid idea and show two possible ions which could be used. In Sec. III we present the different set-ups studied, discussing the choice of boost factor, baseline and various detector options. The description of the simulation and analysis is presented in Sec. IV and the results in Sec. V] The final discussion and conclusions are drawn in Secs. VI and VII.

\section{THE BETA-BEAM AND ELECTRON CAPTURE COMBINATION}

In this section we introduce the idea of the beta-beam and electron capture hybrid approach. We present the spectra of the two branches, their ratio and discuss two nuclides which have desirable properties.

The beta-beam is a proposal, originally put forward by P. Zucchelli [22], to accelerate and then store $\beta$-emitting ions, which subsequently decay to produce a well collimated, uncontaminated, electron neutrino (or antineutrino) beam. The high luminosities required to achieve a useful physics reach point towards ions with small proton numbers to minimise space charge and half-lives $\sim 1$ second to reduce ion losses during the acceleration stage whilst maintaining a large number of useful decays per year. The most promising candidate ions are ${ }^{18} \mathrm{Ne}$ and ${ }^{8} \mathrm{~B}$ for neutrinos, and ${ }^{6} \mathrm{He}$ and ${ }^{8} \mathrm{Li}$ for antineutrinos. A variant on the beta-beam idea is the use of electron capture to produce monoenergetic neutrino beams. Electron capture is the process in which an atomic electron is captured by a bound proton of the ion $A(Z, N)$ leading to a nuclear state of the same atomic number $A$, but with the exchange of the proton by a neutron and the emission of an electron neutrino,

$$
A(Z, N)+e^{-} \rightarrow A(Z-1, N+1)+\nu_{e}
$$

The idea of using this process in neutrino experiments was independently discussed in Refs. [53, 55]. In Ref. [56], ions with low $Q_{\mathrm{EC}}$-value and long half-life, such as ${ }^{110} \mathrm{Sn}$, were proposed to be accelerated to very high boosts with the LHC. Baselines of $250 \mathrm{~km}$ and $600 \mathrm{~km}$ were considered with the spectral information coming from the position of the events in the detector. Sensitivities comparable to a Neutrino Factory were obtained for a 
single boost. However, in order for electron capture machines to become operational, nuclei with shorter half-life are required. The recent discovery of nuclei far from the stability line with kinematically accessible super-allowed spin-isospin transitions to giant Gamow-Teller resonances (see, for example, Ref. [57]) opens up such a possibility. The rare-Earth nuclei above ${ }^{146} \mathrm{Gd}$ have a short enough half-life to allow electron capture processes in the decay ring, in contrast to fully-stripped long-lived ions $[55,56]$. This was the alternative put forward in Ref. [53] where the use of short-lived ions with $Q_{\mathrm{EC}}$-values around $1-4 \mathrm{MeV}$ was proposed. Machines such as the SPS, an upgraded SPS and the Tevatron could then be used for the acceleration. The ion ${ }^{150} \mathrm{Dy}$, with $Q_{\mathrm{EC}}$-value $1.4 \mathrm{MeV}$, was investigated for the CERN-Frejus $(130 \mathrm{~km})$ and CERN-Canfranc $(650 \mathrm{~km})$ baselines and different boost factors. It was found to have very good physics reach $[53,54]$. Owing to the monochromatic nature of the beam, multiple boosts are necessary to resolve the intrinsic degeneracy in this case.

In the following, we demonstrate how the flux for the electron capture/beta-beam can be built up by discussing them separately and comparing branching ratios. Let the mass difference between the parent and the daughter nuclei, $\Delta M_{A}^{\beta^{+}}=M_{A}(Z, N)-M_{A}(Z-$ $1, N+1$ ), include the mass and the binding energy of an atomic electron as well. For electron capture, the maximum kinetic energy release is thus given by $Q_{\mathrm{EC}}=\Delta M_{A}^{\beta^{+}}$. For $\beta^{+}$-decay, however, the final nucleus has an excess electron since a positron is produced. The maximum kinetic energy release is thus given by $Q_{\beta^{+}}=\Delta M_{A}^{\beta^{+}}-2 m_{e}$. Clearly for $\left(\Delta M_{A}^{\beta^{+}}=\right) Q_{\mathrm{EC}}<2 m_{e}$, electron capture is the only allowed process for a proton-rich nucleus. For $Q_{\mathrm{EC}}>2 m_{e}$, electron capture and positron emission compete, their branching ratios dependent on $Q_{\mathrm{EC}}$. If decay through $\alpha$ emission is also allowed, it is important that this has a relatively low $Q$-value so as not to be the dominant channel ${ }^{3}$. For a number of useful ion decays per year $N_{\text {ions }}$, the electron capture neutrino flux is given by [53, 54]

$$
\frac{d \Phi_{\mathrm{EC}}^{\mathrm{lab}}}{d \Omega d E_{\nu}}=\frac{\Gamma}{\Gamma_{\text {tot }}} \frac{N_{\text {ions }}}{\pi L^{2}} \gamma^{2} \delta\left(E_{\nu}-2 \gamma E_{0}^{\mathrm{EC}}\right)
$$

for each decay channel. Here, $L$ is the baseline, $\gamma$ is the Lorentz boost, $E_{0}^{\mathrm{EC}}\left(=Q_{\mathrm{EC}}\right)$ is the neutrino energy in the ion rest frame and $E_{\nu}$ is the neutrino energy in the lab frame.

The flux for the $\beta$-spectrum is found in the usual way. In the rest frame of the ion, the

\footnotetext{
${ }^{3}$ The $\alpha$ decay branching ratio is strongly dependent on the $Q_{\mathrm{EC}}$-value. For low $Q_{\mathrm{EC}}$, the $\alpha$ decay probability is sufficiently long as to allow the weak decay modes to be the main channels.
} 
electron neutrino flux is proportional to

$$
\frac{d \Phi_{\beta}^{\mathrm{rf}}}{d \cos \theta d E_{\mathrm{rf}}} \sim E_{\mathrm{rf}}^{2}\left(E_{0}^{\beta}-E_{\mathrm{rf}}\right) \sqrt{\left(E_{\mathrm{rf}}-E_{0}^{\beta}\right)^{2}-m_{e}^{2}} .
$$

Here, $E_{0}^{\beta}\left(=Q_{\beta^{+}}+m_{e}=Q_{\mathrm{EC}}-m_{e}\right)$ is the total end-point energy of the decay. The neutrino flux per solid angle at the detector located at distance $L$ from the source after boost $\gamma$ is [48]

$$
\left.\frac{d \Phi_{\beta}^{\mathrm{lab}}}{d \Omega d y}\right|_{\theta \simeq 0} \simeq \frac{N_{\text {ions }}}{\pi L^{2}} \frac{\gamma^{2}}{g\left(y_{e}\right)} y^{2}(1-y) \sqrt{(1-y)^{2}-y_{e}^{2}},
$$

where $0 \leq y=\frac{E_{\nu}}{2 \gamma E_{0}^{\beta}} \leq 1-y_{e}, y_{e}=m_{e} / E_{0}^{\beta}$, and

$$
g\left(y_{e}\right) \equiv \frac{1}{60}\left\{\sqrt{1-y_{e}^{2}}\left(2-9 y_{e}^{2}-8 y_{e}^{4}\right)+15 y_{e}^{4} \log \left[\frac{y_{e}}{1-\sqrt{1-y_{e}^{2}}}\right]\right\}
$$

Similarly to the case of electron capture, a neutrino with energy $E_{\mathrm{rf}}$ in the rest frame will have a corresponding energy $E_{\nu}=2 \gamma E_{\mathrm{rf}}$ in the laboratory frame along the $\theta=0^{\circ}$ axis.

All the known nuclear structure information on the $A=148$ and $A=156$ nuclides has been reviewed in Ref. [58] and Ref. [59], respectively, where the information obtained in various reaction and decay experiments is presented, together with adopted level schemes. Currently, a systematic study of electron capture decays in the region of ${ }^{146} \mathrm{Gd}$, relevant for monoenergetic neutrino beams, is being carried out [60]. Here, we consider two nuclides, ${ }_{70}^{156} \mathrm{Yb}$ and ${ }_{65}^{148 m} \mathrm{~Tb}$, that decay through electron capture and $\beta^{+}$-decay with similar branching ratios whose lifetimes are not too long or too short. Their decays are summarised in Tables [ and II]. Ytterbium is a nuclide ${ }_{70}^{156} \mathrm{Yb}$ with spin-parity $0^{+}$, which decays $90 \%$ via electron capture plus $\beta^{+}$-decay [59], with $38 \%$ via electron capture and $52 \%$ via $\beta^{+}$-decay [60]. The remaining $10 \%$ goes into alpha particles and a different final state. This relatively small branching ratio into alphas helps the nuclide to have a short enough half-life, 26.1 seconds. It is important to note that this electron capture- $\beta^{+}$-decay transition has only one possible daughter state with spin-parity $1^{+}$, i.e., it is a Gamow-Teller transition into an excited state

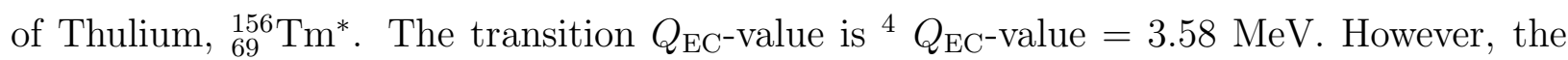
excitation energy of the final nuclear state $(0.12 \mathrm{MeV})$ needs to be taken into account and thus, the effective $Q_{\mathrm{EC}}$-value (difference in the total kinetic energies of the system after and before the decay) is $3.46 \mathrm{MeV}$ [59]. The electron capture energy of $\sim 4 \mathrm{MeV}$ is well suited

\footnotetext{
${ }^{4} Q_{\mathrm{EC}}$-values are typically calculated between ground states unless stated otherwise.
} 


\begin{tabular}{|c|c|c|c|}
\hline Decay & Daughter & Neutrino energy (MeV) & BR \\
& & & \\
\hline \hline$\beta^{+}$ & ${ }^{156} \mathrm{Tm}^{*}$ & 2.44 (endpoint) & $52 \%$ \\
$\mathrm{EC}$ & ${ }^{156} \mathrm{Tm}^{*}$ & 3.46 & $38 \%$ \\
$\alpha$ & ${ }^{152} \mathrm{Er}$ & 4.81 & $10 \%$ \\
\hline
\end{tabular}

TABLE I: Decay summary for ${ }_{70}^{156} \mathrm{Yb}$. The $Q_{\mathrm{EC}}$-value for the transition between ground states is $3.58 \mathrm{MeV}$ and taking into account the excitation energy of the final nuclear state $(0.12 \mathrm{MeV})$, the effective $Q_{\mathrm{EC}}^{\mathrm{eff}}$-value is $3.46 \mathrm{MeV}[59,60]$.

\begin{tabular}{|c|c|c|c|}
\hline Decay & Daughter & Neutrino energy (MeV) & BR \\
\hline \hline$\beta^{+}$ & ${ }_{64}^{148} \mathrm{Gd}^{*}$ & 2.05 (endpoint) & $32 \%$ \\
EC & ${ }_{64}^{148} \mathrm{Gd}^{*}$ & 3.07 & $68 \%$ \\
\hline
\end{tabular}

TABLE II: Decay summary for ${ }_{65}^{148 m} \mathrm{~Tb}$. The $Q_{\mathrm{EC}}$-value for the transition between ground states is $5.77 \mathrm{MeV}$ and the effective $Q_{\mathrm{EC}}^{\mathrm{eff}}$-value to the excited state is $3.07 \mathrm{MeV}[58,61,62]$.

to the intermediate-baselines of Europe and the USA with the available technology, or those available with future upgrades. On the other hand, the ${ }_{65}^{148 m} \mathrm{~Tb}$ isomer with spin-parity $9^{+}$ has a $Q_{\mathrm{EC}}$-value of $5.77 \mathrm{MeV}[58,61]$. Although the decay to the ground state of ${ }_{64}^{148} \mathrm{Gd}$ is highly forbidden, the presence of a Gamow-Teller resonance allows the decay into an excited state with effective $Q$-value $3.07 \mathrm{MeV}$ [62]. This nuclide is longer lived than ${ }_{70}^{156} \mathrm{Yb}$ (with a half-life of 2.2 minutes) and will require slightly higher boosts. It is still well suited to intermediate baselines. However, the dominance of the electron capture over the $\beta^{+}$-decay channel makes this nuclide less desirable. The count rate will be dominated by the single energy of the electron capture which provides insufficient information to obtain the good sensitivities aspired to by future long baseline experiments. It was shown in Refs. [53, 54] that two runs with different boosts are necessary for an exclusive or dominant electron capture channel to break the intrinsic degeneracy and achieve good CP-violation discovery. Hence, in what follows we will study this hybrid approach focusing on ${ }^{156} \mathrm{Yb}$. 


\section{EXPERIMENTAL SETUPS}

In this section we consider different boosts, baselines and detectors. We first discuss the available (or possible future) accelerator technology and identify the possible boost factors in combination with different baselines. We then discuss the main characteristics of the detectors considered in the analysis.

\section{A. Choice of $\gamma$ and baseline}

In this paper we consider the use of a neutrino beam sourced from boosted ${ }^{156} \mathrm{Yb}$ ions directed along a single baseline. As described above, both the electron capture and $\beta^{+}$-decay channels are to an excited state of ${ }^{156} \mathrm{Tm}$ with a $Q_{\mathrm{EC}}$-value of $3.46 \mathrm{MeV}$. In order to fully exploit the electron capture decay mode, the nuclides cannot be fully stripped; at least 16 electrons being left on the ion [63]. The maximum boost, $\gamma_{\max }$, available is thus

$$
\gamma_{\max }=\frac{E_{\mathrm{acc}}}{m_{p}} \frac{Z-16}{A},
$$

where $m_{p}$ is the mass of the proton and $E_{a c c}$ is the maximum energy accessible with the accelerator. Current and future accelerator facilities would be an ideal production environment. In this analysis, we consider the maximum boosts available from the current SPS and upgraded SPS (see Table III) for the following baselines:

\section{Boost $\gamma=166$ with current SPS}

- CERN-Frejus (130 km)

- CERN-Canfranc $(650 \mathrm{~km})$

\section{Boost $\gamma=369$ with an upgraded SPS}

- CERN-Canfranc $(650 \mathrm{~km})$

- CERN-Boulby $(1050 \mathrm{~km})$

With the current magnetic rigidity of the SPS, the electron capture spike can be placed on first oscillation for the CERN-Canfranc baseline $(650 \mathrm{~km})$ with the beta-beam spectrum peaking around the second oscillation maximum (see Fig. 1). 

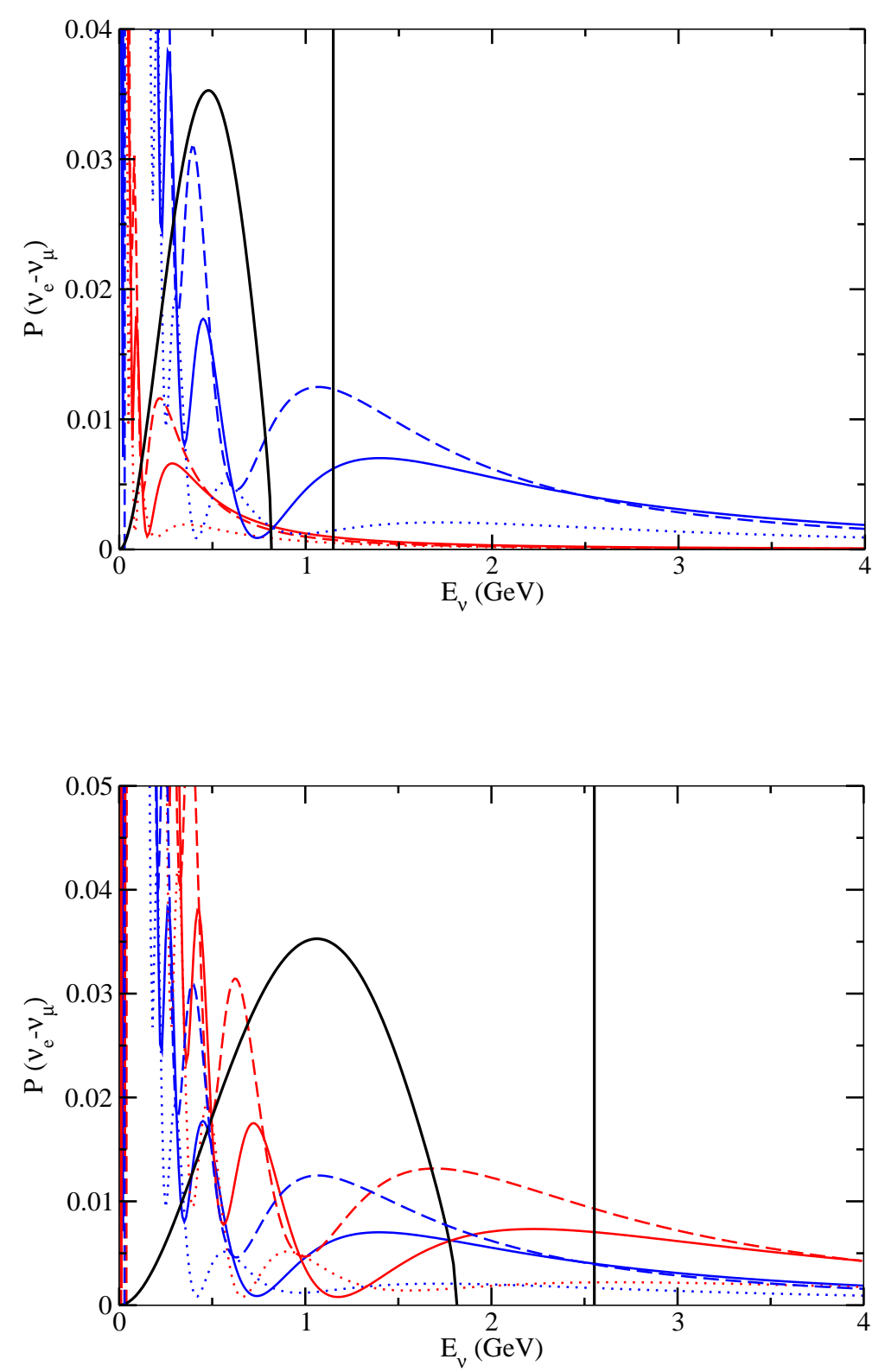

FIG. 1: Top panel: $\nu_{e} \rightarrow \nu_{\mu}$ appearance probabilities for the CERN-Frejus (130 km) and CERNCanfranc $(650 \mathrm{~km})$ baselines. The unoscillated $\nu_{e}$ flux in the laboratory frame is shown for ${ }^{156} \mathrm{Yb}$ given a boost $\gamma=166$ in arbitrary units. Bottom panel: $\nu_{e} \rightarrow \nu_{\mu}$ appearance probabilities for the CERN-Canfranc $(650 \mathrm{~km})$ and CERN-Boulby $(1050 \mathrm{~km})$ baselines. The flux from a boost $\gamma=369$ is shown in arbitrary units. In both cases, the blue lines correspond to CERN-Canfranc; the red being CERN-Frejus (top panel) and CERN-Boubly (bottom panel). The solid lines correspond to $\delta=0^{\circ}$, dashed $\delta=90^{\circ}$ and dotted $\delta=-90^{\circ}$. The value $\sin ^{2} 2 \theta_{13}=0.01$ was taken for all curves. 


\begin{tabular}{|c|c|c|c|}
\hline Machine & $\gamma_{\max }$ & $2 \gamma_{\max } Q_{\mathrm{EC}}^{\mathrm{eff}}(\mathrm{GeV})$ & $2 \gamma_{\max } Q_{\beta^{+}}^{\mathrm{eff}}(\mathrm{GeV})$ \\
\hline \hline SPS & 166 & 1.15 & 0.81 \\
Upgraded SPS & 369 & 2.55 & 1.80 \\
\hline
\end{tabular}

TABLE III: Maximum boosts and neutrino endpoint energies for ${ }^{156} \mathrm{Yb}$ available for the current SPS setup and a proposed $1 \mathrm{GeV}$ upgraded SPS.

A detector with a low energy threshold is necessary to exploit the oscillatory structure of the appearance probability. The second option would make use of the upgrades to the CERN accelerator facilities necessary for suggested LHC upgrades. With a 1 TeV SPS, the electron capture beam could be placed at first oscillation maximum, or on the probability tail, for the CERN-Boulby baseline $(1050 \mathrm{~km})$ or, using the Tevatron, for the FNAL-Homestake baseline $(1280 \mathrm{~km})$. With an energy threshold of $250 \mathrm{MeV}$, these setups could exploit the information at second oscillation maximum to resolve some of the degeneracies in an approach analogous to Ref. [29], where the boost for ${ }^{18} \mathrm{Ne}$ was chosen so that the boosted spectrum covered both first and second maximum. Owing to the spectral nature of the decay, in the analysis in Ref. [29] it was difficult to determine to what extent the highest energies contribute to the overall sensitivity of the setup. With the electron capture beta-beam combination we can investigate this issue as it is possible to place the electron capture spike on first oscillation maximum or on its tail whilst the beta-beam spectrum has minimal coverage of that energy range. In our analysis we perform $\left(\theta_{13}, \delta\right)$ sensitivity contours through the consideration of each decay channel separately and their combination. This allows us to investigate the importance of the contribution of each decay channel and evaluate if the importance of the low and high energy contributions to the overall sensitivity.

\section{B. Choice of detector}

In a beta-beam, one aims to exploit the $\nu_{e} \rightarrow \nu_{\mu}$ channel; a detector with excellent muon identification capabilities and efficient neutral current background rejection is therefore required. For energies below $\sim 1 \mathrm{GeV}$, water-Čerenkov detectors are typically chosen with the muons identified through the use of quasi-elastic events (QE). Efficient reduction of neutral current events and (subdominant) pions is through the identification of the decay 
process. For higher energies, the number of QE events drops sharply where the deep inelastic scattering (DIS) component dominates the cross section. Water-Čerenkov detectors are usually not the best choice for the higher energies owing to high backgrounds and poor neutrino energy resolution. For large boosts, the 50 kton class of detectors such as liquid argon (LAr), using time projection chamber techniques, or total active scintillator detector (TASD), based on tracking calorimeter principles, are usually considered. In addition to QE events, these technologies also measure the energy deposited through the hadronic channels and DIS events are in principle also distinguishable. Their main disadvantage is their size which is far smaller than the next generation water-Čerenkov detectors discussed in the literature which typically have fiducial masses in the megaton scale.

For a pure electron capture machine, the choice of detector technology does not depend on its energy reconstruction capabilities. In this case, the neutrino energy is given by the choice of ion and boost factor leaving no need to reconstruct the neutrino energy in the detector. For the hybrid approach we consider, it is possible, in principle to separate the energy of the line spectrum from the continuous spectrum. Suppose we identify an event and classify it as being a $\mathrm{QE}$ elastic event with energy $E_{\nu}(\mathrm{QE})$, then it must be the case that the true energy $E_{\nu}^{\text {true }} \geq E_{\nu}(\mathrm{QE})$. Thus, if one measures $E_{\nu}(\mathrm{QE})>2 \gamma E_{0}^{\beta}$, then this event must be attributed to the electron capture flux and hence, it is not necessary to reconstruct more precisely the true neutrino energy. The separation between the energy of the electron capture spike and the end-point energy of the beta-spectrum is $2 m_{e} \gamma$. This should render the distinction between the electron capture and the beta-beam fluxes possible. We will assume this throughout the paper.

In the analysis, we will follow two strategies regarding the detector type. We consider a generic detector technology, which could be LAr or TASD, with a fiducial mass of 50 kton and assume that the neutrino spectral information can be extracted from the charged current events. On the other hand we also consider a 0.5 Mton (fiducial) water-Čerenkov detector. In this case, following the prescription described above, we assume the neutrino energy from beta-beam events can only be reconstructed for QE events. However, we do include the information from the inelastic events. As no spectral information is possible for those events, we include them in a single bin. We will take perfect efficiency for the 50 kton detector (which can be easily scaled), and $70 \%$ efficiency for the water-Čerenkov detector. 


\section{SIMULATION AND ANALYSIS}

Based on the selected boost factors, baselines and type of detector we have discussed in detail in the previous section, in our analysis we will study and compare six different setups:

1. 50 kton detector (LAr or TASD) with $2 \times 10^{18}$ ions $/ y r$

- Setup I: CERN-Frejus (130 km) and $\gamma=166$

- Setup II: CERN-Canfranc $(650 \mathrm{~km})$ and $\gamma=166$

- Setup III: CERN-Canfranc $(650 \mathrm{~km})$ and $\gamma=369$

- Setup IV: CERN-Boulby $(1050 \mathrm{~km})$ and $\gamma=369$

\section{0.5 Mton water-Čerenkov detector with $2 \times 10^{18}$ ions $/ y r$}

- Setup III-WC: CERN-Canfranc $(650 \mathrm{~km})$ and $\gamma=369$

- Setup IV-WC: CERN-Boulby $(1050 \mathrm{~km})$ and $\gamma=369$

We will take a running time of 10 years for all the experimental configurations considered.

The number of events is computed for each energy bin $i$, given by

$$
n_{i}=\mathcal{N} \int d E \Phi(E) P(E) \sigma(E) K_{i}(E)
$$

where $\mathcal{N}$ is a constant which takes into account the efficiency, the mass of the detector and the running time, $\Phi(E)$ is the neutrino flux spectrum at the detector, $P(E)$ is the probability function, $\sigma(E)$ represents the total, QE or DIS cross section (as described in the previous section) and $K_{i}(E)$ is the energy smearing kernel for the $i$ th bin for which we take a Gaussian energy resolution function with a constant width of $150 \mathrm{MeV}$.

In the experimental simulations performed in this study, our analysis is based on the following $\chi^{2}$ definition

$$
\chi^{2}=\sum_{i, j}\left(n_{i}^{t}-n_{i}^{f}\right) C_{i j}^{-1}\left(n_{j}^{t}-n_{j}^{f}\right),
$$

where $n_{i}^{f}$ are the predicted (or fitted) number of events for a certain oscillation hypothesis, and $n_{i}^{t}$ are the simulated "data" for the true values of the parameters. The covariance matrix $C$ given by

$$
C_{i j}=\delta_{i j}\left(\delta n_{i}^{t}\right)^{2}
$$


where $\left(\delta n_{i}^{t}\right)=\sqrt{n_{i}^{t}+\left(f_{\text {sys }} \cdot n_{i}^{t}\right)^{2}}$, contains both statistical and a $2 \%$ overall systematic error $\left(f_{\text {sys }}=0.02\right)$. In addition, we assume an intrinsic beam background of $0.1 \%$ of the unoscillated spectrum originating from neutral current pion production and muons misidentified as electrons. In the energy range of interest, there are about 30 atmospheric neutrino events per kton-year which could mimic a muon coming from a $\nu_{e} \rightarrow \nu_{\mu}$ oscillation. We take $10^{-3}$ as the accelerator duty factor so that this atmospheric background amounts to 0.03 events per kton-year.

In all setups considered in this paper, for the beta-beam part we take an energy threshold of $250 \mathrm{MeV}$ and use $200 \mathrm{MeV}$ bins above that value, except for non-QE events in waterČerenkov detectors which are grouped in a single bin. For the electron capture events we always take a single bin. Unless otherwise stated, we impose restrictions on certain subsets of the fitted parameters in order to account for external information from other experiments. Thus, we introduce the so-called priors. Hence, if we want to restrict some parameter $\kappa$, we introduce the central value $\kappa_{c}$ of the prior and the input error $\sigma_{\kappa}$, and the actual minimisation is performed over the modified $\chi^{2}$ function

$$
\chi^{2} \rightarrow \chi^{2}+\frac{\left(\kappa-\kappa_{c}\right)^{2}}{\sigma_{\kappa}^{2}} .
$$

In this work, we set priors for the experimentally known oscillation parameters, taking their best fit values as central values [11] of the corresponding priors, and the half width of one standard deviation of their best fit values as the corresponding input errors. Specifically, $\Delta m_{21}^{2},\left|\Delta m_{31}^{2}\right|, \sin ^{2} \theta_{12}$ and $\sin ^{2} \theta_{23}$ have been given errors $3 \%, 5 \%, 7 \%$ and $14 \%$ respectively. We marginalise over $\theta_{13}$ and $\delta$ over their entire range.

\section{RESULTS}

In this section we present and discuss the results of our detailed numerical analysis of the various setups. In order to understand some of the features of these results, it is useful to consider an analytical approximation for the oscillation probability which for these energies and baselines is given by [64] (see also Ref. [65]):

$$
\begin{aligned}
& P\left(\nu_{e} \rightarrow \nu_{\mu}, L\right) \simeq \sin ^{2} \theta_{23} \sin ^{2} 2 \theta_{13}\left(\frac{\Delta_{13}}{A-\Delta_{13}}\right)^{2} \sin ^{2}\left(\frac{\left(A-\Delta_{13}\right) L}{2}\right) \\
& \quad+\cos \theta_{13} \sin 2 \theta_{13} \sin 2 \theta_{23} \sin 2 \theta_{12} \frac{\Delta_{12}}{A} \frac{\Delta_{13}}{A-\Delta_{13}} \sin \left(\frac{A L}{2}\right) \sin \left(\frac{\left(A-\Delta_{13}\right) L}{2}\right) \cos \left(\frac{\Delta_{13} L}{2}+\delta\right) \\
& \quad+\cos ^{2} \theta_{23} \sin ^{2} 2 \theta_{12}\left(\frac{\Delta_{12}}{A}\right)^{2} \sin ^{2}\left(\frac{A L}{2}\right),
\end{aligned}
$$


where $\Delta_{12} \equiv \Delta m_{21}^{2} /(2 E)$ and $\Delta_{13} \equiv \Delta m_{31}^{2} /(2 E)$. We use the constant density approximation for the index of refraction in matter $A \equiv \sqrt{2} G_{F} \bar{n}_{e}(L)$, with $\bar{n}_{e}(L)=1 / L \int_{0}^{L} n_{e}\left(L^{\prime}\right) d L^{\prime}$ the average electron number density. We analyse the sensitivity to $\theta_{13}$ and $\delta$ for all the setups, and discuss the discovery reach for CP-violation and the type of neutrino mass ordering.

\section{A. Sensitivity to $\theta_{13}$ and $\delta$}

\section{Setups I and II}

Setups I and II use a low boost factor, $\gamma=166$ and a 50 kton detector, implying relatively low count rates. We find that the sensitivity to $\theta_{13}$ and $\delta$ is very limited as a consequence. Violation of $\mathrm{CP}$ can be established only for a small range of values of the $\delta$ phase and only if $\theta_{13}$ is close to the present bounds. The beta-beam channel contributes very little to the overall sensitivity of the setup. This is due to the $\gamma^{2}$-dependence of a beta-beam flux. The small flux, when combined with the small cross-sections at the energies centred on second oscillation maximum, supplies a scarce count rate. The bulk of the sensitivity is therefore due to the electron capture channel placed on first oscillation maximum, as seen in Fig. 2 , The performance of the beta-beam (upper row), electron capture (middle row) and their combination (lower row) is shown for $\theta_{13}=1^{\circ}$ and $\delta=90^{\circ}$ for setup I (left column) and setup II (right column), at 90\%, 95\% and 99\% confidence level (CL).

\section{Setups III and III-WC}

We have also examined the effect of placing the electron capture beam in the tail of first oscillation maximum. This gives the beta-beam coverage of the second oscillation maximum and substantial portions of the first oscillation maximum. For the CERN-Canfranc baseline and a boost $\gamma=369$, we find that the roles of electron capture and the beta-beam are reversed compared to $\gamma=166$. The beta-beam spectrum is peaked around $\sim 1 \mathrm{GeV}$ while the EC energy is of $2.55 \mathrm{GeV}$. As shown in Fig. 1, the neutrino beta-beam spectrum is a good fit for the appearance probability at Canfranc, its peak sitting around the first oscillation maximum. The beta-beam now contributes much more to the sensitivity as it provides substantial information from the first oscillation maximum and a much higher count rate from the second oscillation maximum.

In Fig. 3 and Fig. 4 we show the 90\%, 95\% and 99\% CL contours for the setup III and 

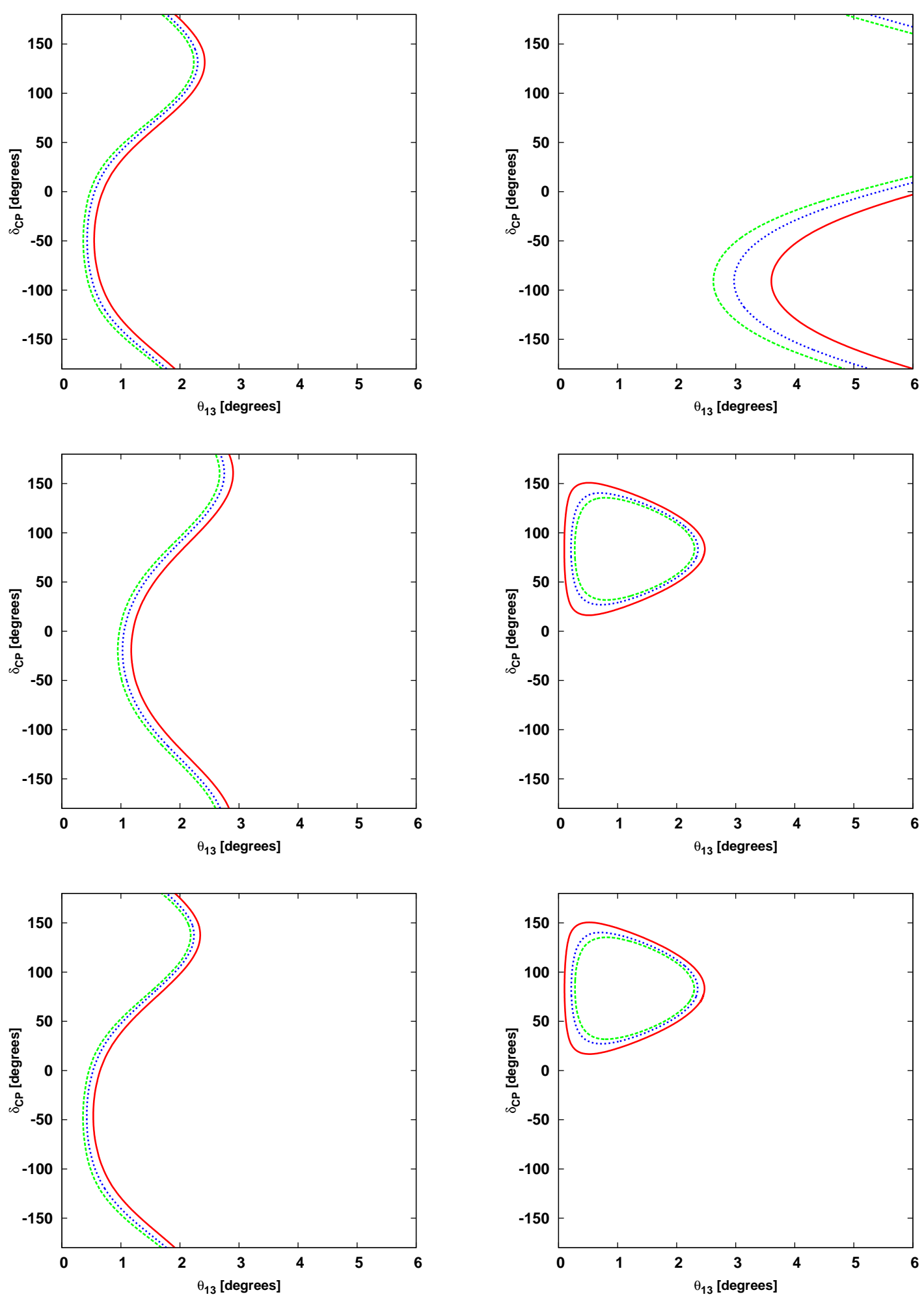

FIG. 2: 90\%, 95\% and 99\% CL contours for setup I (left panels) and setup II (right panels). The parameters $\theta_{13}=1^{\circ}$ and $\delta=90^{\circ}$ have been taken assuming normal mass ordering and $\theta_{23}=45^{\circ}$. The upper row is the contribution of the beta-beam, the middle row is that of the electron capture channel with the lower row being the combination. 
setup III-WC, respectively, assuming that the hierarchy is normal and $\theta_{23}=45^{\circ}$. In Fig. 3, we present contours for $\delta=90^{\circ}$ and for the cases $\theta_{13}=1^{\circ}$ (left column) and $3^{\circ}$ (right column). Similarly to Fig. 2, in Fig. 3, we have included the contributions from both the $\beta^{+}$-decay (upper row) and electron capture (middle row) channels separately to investigate their relative impact, in addition to the total sensitivity (lower row).

Fig. 4 shows the results for $\theta_{13}=1^{\circ}$ (left column) and $3^{\circ}$ (right column) and four values for $\delta$, including the hierarchy clone solution. Comparing the results for the setup III and setup III-WC, one can understand the effect of the event rate. We see that the 0.5 Mton WC detector gives a much better resolution, although not as much as one would naïvely expect from its larger size. As commented above, the substantially bigger size of the detector cannot be fully exploited due to the fast drop of the QE cross section at energies above $1 \mathrm{GeV}$, where the first oscillation maximum lies.

The full power of the combination between the beta-beam spectrum and the EC channel is best illustrated in Fig. 3. We see that each of the two techniques separately suffer from a continuum of solutions. The shape of the allowed region in the $\theta_{13}$ and $\delta$ plane can be easily understood by looking at the form of the oscillation probability in Eq. (5.1). Owing to the relatively short baseline and small matter effects, we can neglect for simplicity the matter potential term, $A$, in the following. As we measure the probability in only one polarity, for fixed energy and baseline, as is the case for the EC signal, we can relate the allowed values of $\delta$ and $\theta_{13}$ from a measurement of the probability as

$$
\sin 2 \theta_{13} \simeq-\frac{\Delta_{21}}{\Delta_{31}} \frac{\Delta_{31} L}{\sin \left(\Delta_{31} L / 2\right)} \cos \left(\frac{\Delta_{13} L}{2}+\delta\right)+k
$$

where $k$ is a constant which depends on the true values of $\theta_{13}$ and $\delta$. This approximation is valid as far as $\sin ^{2} 2 \theta_{13} \gg$ few $\times 10^{-3}$. The form of the expression in Eq. (5.2) matches the continuum of solutions in Fig. 3. In particular, we note the presence of a minimum for $\theta_{13}$ at $\delta=-\Delta_{13} L / 2$. This result is more general then the approximated form of $\sin \theta_{13}$ in Eq. (5.2) and holds also for small values of $\sin \theta_{13}$. For the choice of the parameters used in Fig. 3, we have $\delta \simeq-44^{\circ}$. The range of the allowed solutions for $\theta_{13}$ can be computed by looking at the amplitude of the function in Eq. 5.2 and is found to be $\Delta \theta_{13} \sim \frac{1}{2} \frac{\Delta_{21}}{\Delta_{31}} \frac{\Delta_{31} L / 2}{\sin \left(\Delta_{31} L / 2\right)}$. For the choice of true values in Fig. [3, $\Delta \theta_{13} \sim 2^{\circ}$.

Now consider the contribution of the beta-beam. For simplicity, we analyse its impact by looking at the energy of the first oscillation maximum, again neglecting matter effects. 

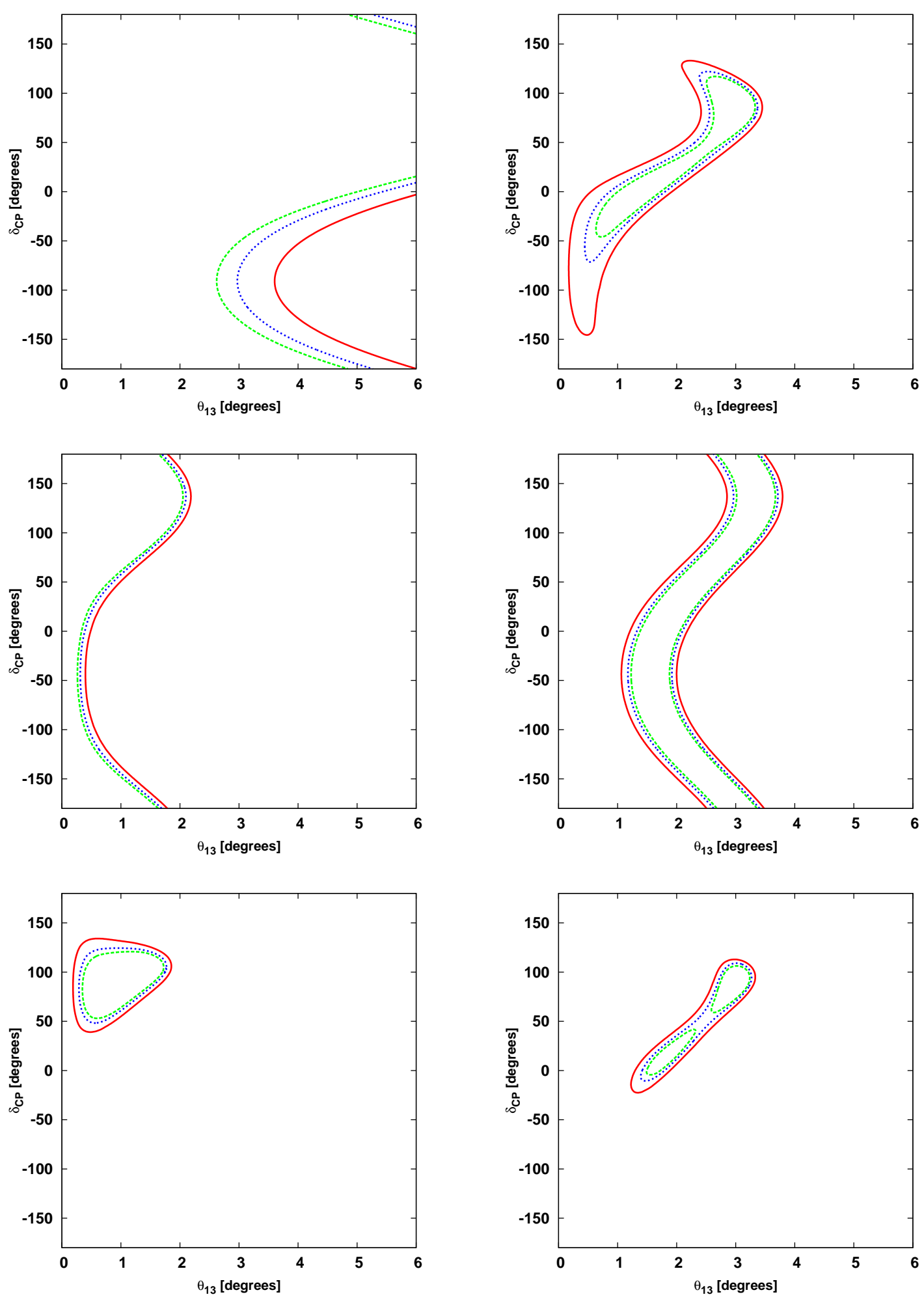

FIG. 3: $90 \%, 95 \%$ and $99 \%$ CL contours for the setup III. The left column is simulated for $\theta_{13}=1^{\circ}$ and $\delta=90^{\circ}$ assuming normal mass ordering and $\theta_{23}=45^{\circ}$. The right column is the same but for $\theta_{13}=3^{\circ}$. The upper row is the contribution of the beta-beam, the middle row is the electron capture channel with the lower row being the combination. 

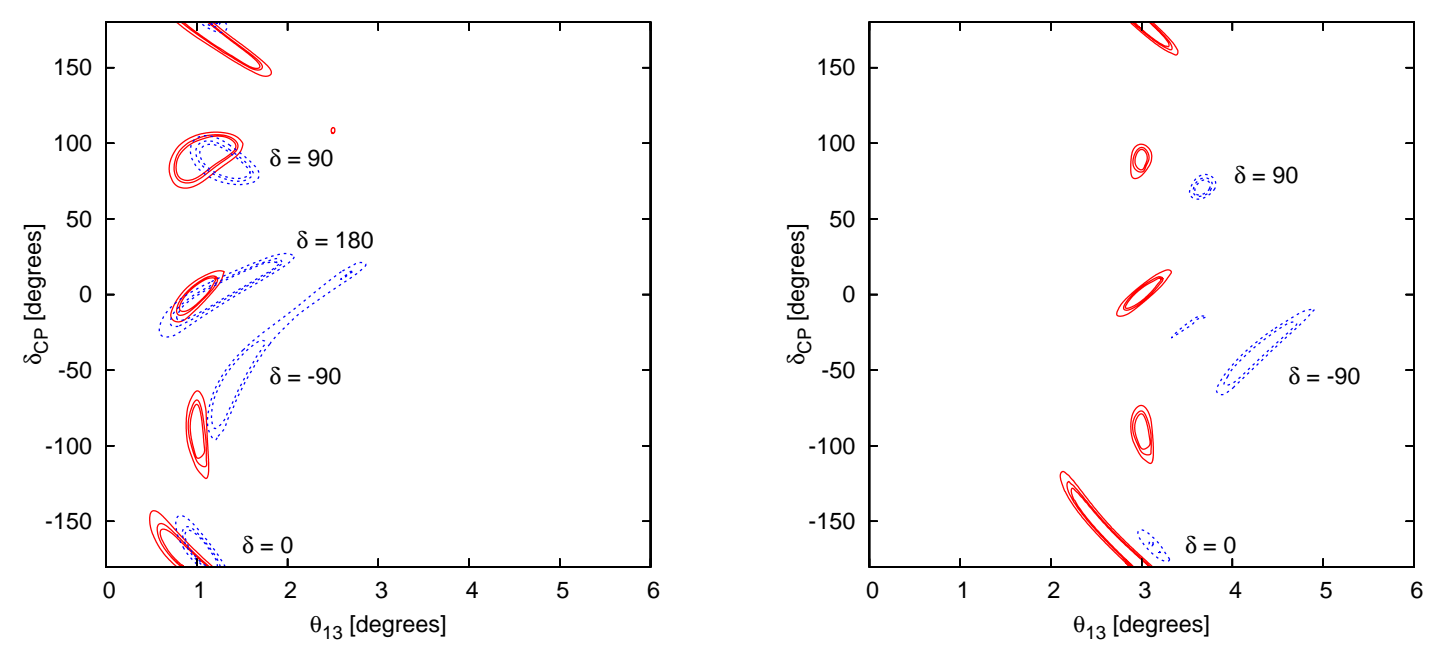

FIG. 4: 90\%, 95\% and 99\% CL contours for setup III-WC with solutions from discrete degeneracies included for $\theta_{13}=1^{\circ}$ (left panel) and $\theta_{13}=3^{\circ}$ (right panel) for different values of the CP-phase, $\delta=-90^{\circ}, 0^{\circ}, 90^{\circ}, 180^{\circ}$.

As for the previous case, the minimum of the continuum solutions for $\theta_{13}$ is located at $\delta=-\Delta_{13} L / 2=-\pi / 2$; shown clearly in Fig. 3 .

The power of the combination of the beta-beam and electron-capture channels is in the difference in phase and in amplitude between the two fake sinusoidal solutions; their combination selects a narrow allowed region in the parameter space, much more constrained then the two separate techniques. This effect is clearly visible in the lower plots in Fig. 3 .

For $\theta_{13}=3^{\circ}$, there is still some intrinsic degeneracy that cannot be completely removed at 99\% CL. The marked difference between the beta-beam alone and the combination with the electron capture in this case demonstrates the importance of data from the high energies. For $\sin ^{2} 2 \theta_{13} \sim 10^{-3}$ and $\delta \sim \pi / 2$, the beta-beam configuration is able to determine the allowed region in the $\left(\theta_{13}-\delta\right)$ parameter space with relatively good accuracy. For this range of $\theta_{13}$, the dominant interference term helps in resolving any degeneracy. This is not the case for other values of $\delta$.

In Fig. 4, we show the results for setup III-WC, with the effects of the hierarchy clone solution taken into account. From a comparison of Figs. 3 and 4, the increase in event rates improves the results substantially. However, owing to the relatively short distance, $L=650 \mathrm{~km}$, the mass ordering can be determined only for large values of the mixing angle 
$\theta_{13}$ (see below). The hierarchy degeneracy worsens the ability to measure $\theta_{13}$ and $\delta$ with good precision, especially for negative true values of $\delta$.

\section{Setups IV and IV-WC}

The use of Boulby instead of Canfranc for a boost of $\gamma=369$ serves as an intermediate case with respect to the last two setups in the sense that the position of the electron capture is neither on first oscillation maximum or far into the tail. Consequently, the beta-beam has some, but not a considerable coverage of the first oscillation region. Boulby provides a much longer baseline, $L=1050 \mathrm{~km}$. This has two contrasting effects on the sensitivity to measure CP-violation: on one side it provides sufficient matter effects to resolve the hierarchy degeneracy even for small values of $\theta_{13}$; on the other, it decreases the available statistics with respect to Canfranc.

In Fig. 5 and Fig. 6, we report the results for setup IV and setup IV-WC, respectively, for normal mass hierarchy and for the cases $\theta_{13}=1^{\circ}$ (left panels) and $\theta_{13}=3^{\circ}$ (right panels). In Fig. [5, we show contours for $\delta=90^{\circ}$. Similarly to Figs. 2 and 3 , in Fig. 5 we present the results for the $\beta^{+}$-decay flux only (upper row), electron capture flux only (middle row) and their combination (lower row). Fig. [6 shows the results for four values for $\delta$, including the hierarchy clone solution (blue dashed contours). The electron capture channel displays similar behaviour to the Canfranc high boost case (setup III, middle row of Fig. (3) at $\theta_{13}=3^{\circ}$. The minimum value of the continuum solutions of $\theta_{13}$ is located at $\delta=-66^{\circ}$ and the allowed range of values is $\sim 2^{\circ}$. For the beta-beam (upper rows), the first oscillation maximum energy bin contributes only marginally to the overall sensitivity, as can be understood from Fig. 1. Therefore, the allowed region in Fig. 5 has a different shape with respect to the case shown in Fig. 3, In addition, the smaller count rate results in a poorer resolution. However, the synergy between beta-beam and electron capture is more important here. The lack of concurrence of the beta-beam and electron capture allowed regions implies that their combination constrains $\delta$ and $\theta_{13}$ in small ranges. This can be clearly seen in Fig. 5 .

In addition, the longer baseline allows for a good determination of the mass ordering (see below), eliminating more degenerate solutions and providing an improved sensitivity to CP-violation with respect to setups III and III-WC. Comparing Fig. [6 with the lower row of Fig. 或, the improvement from setup IV to setup IV-WC is noticeable. 

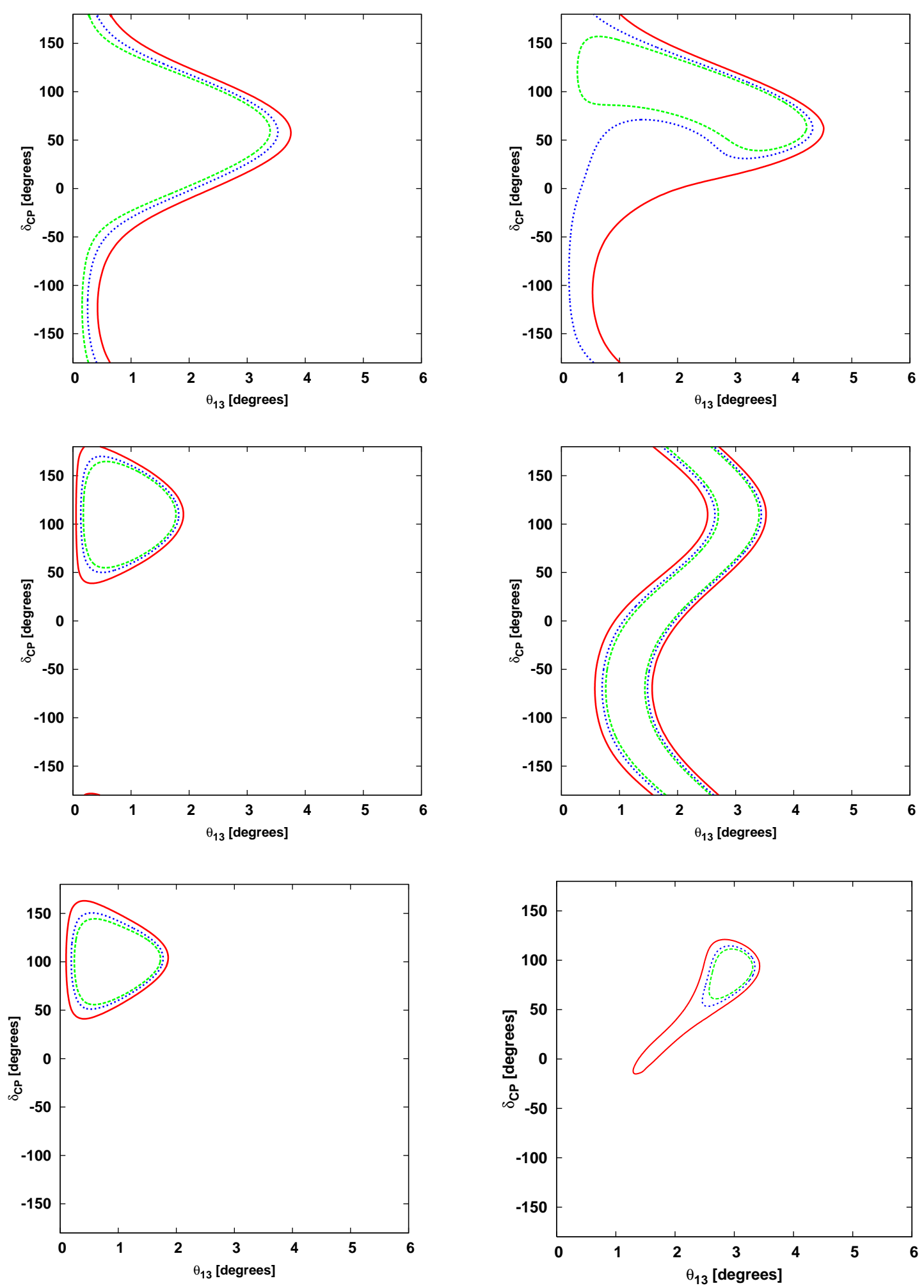

FIG. 5: Same as Fig. 3 but for setup IV. 

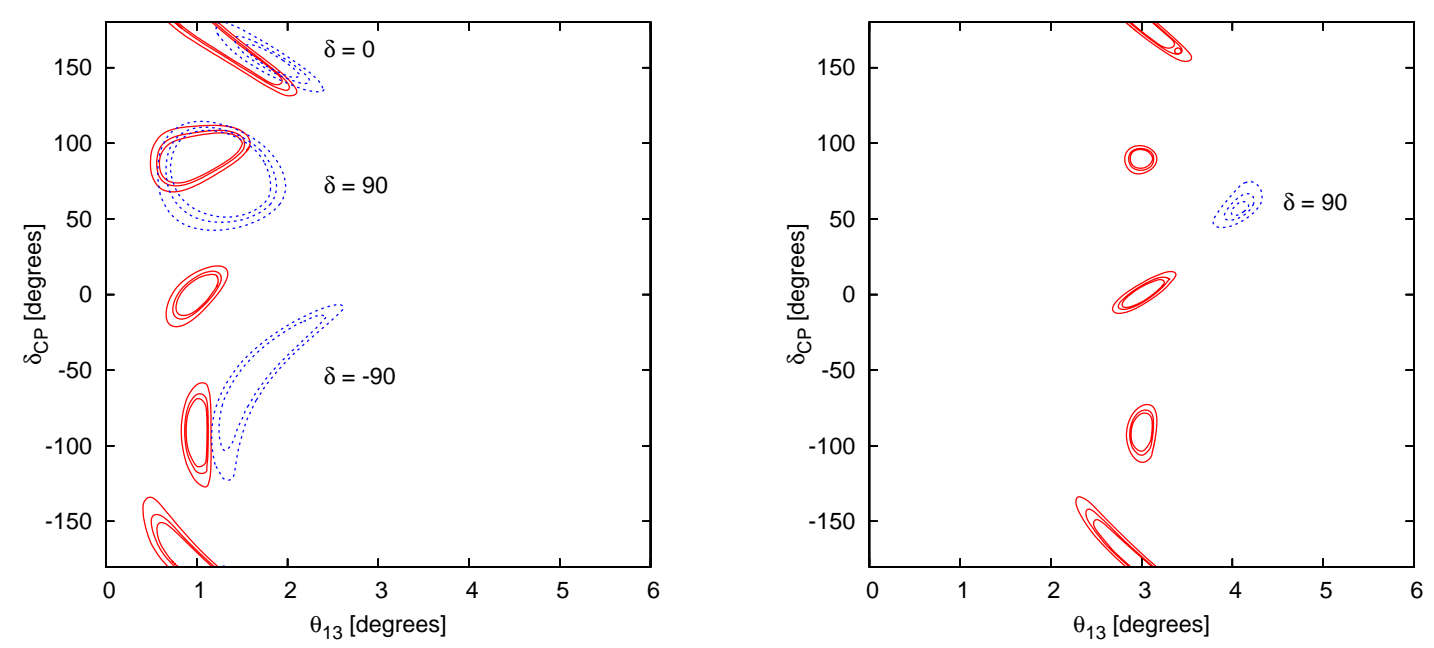

FIG. 6: Same as Fig. 4 but for setup IV-WC.

\section{B. CP-violation discovery potential}

We now consider the CP-discovery potential for the various setups. For the low- $\gamma$ options, setups I and II, the sensitivity is very limited, in agreement with the findings already reported in Fig. 2, Henceforth, we will not show results of these two setup as they always possess worse physics reach compared to the other setups.

Setups III, III-WC, IV and IV-WC have a much better physics reach, as shown in Fig. 7 and Fig. 8, where the CP-violation discovery potential at 99\% CL for the 50 kton TASD or LAr detector (setups III and IV) and 0.5 Mton WC detector (setups III-WC and IV-WC) are depicted, respectively. In both figures, the CERN-Canfranc baseline is displayed in the left panel and the CERN-Boulby baseline in the right panel. In each panel, we present the results for the beta-beam only (blue dotted lines) and the combination with the electron capture result (red solid lines), both without (thin lines) and with (thick lines) taking the hierarchy degeneracy into account. In all cases we note that the addition of the EC channel weakens the impact of the intrinsic degeneracy, significantly improving the sensitivity. The CP-discovery potential depends on various factors, mainly the available count rate and the presence of the hierarchy clone degeneracy. The count rates are important, as can be understood by comparing the results for the setups III with III-WC and IV with IV-WC. However, it should be pointed out that the WC detector is not optimised for the high energies, where the QE cross section is small and the information on the energy for the total 

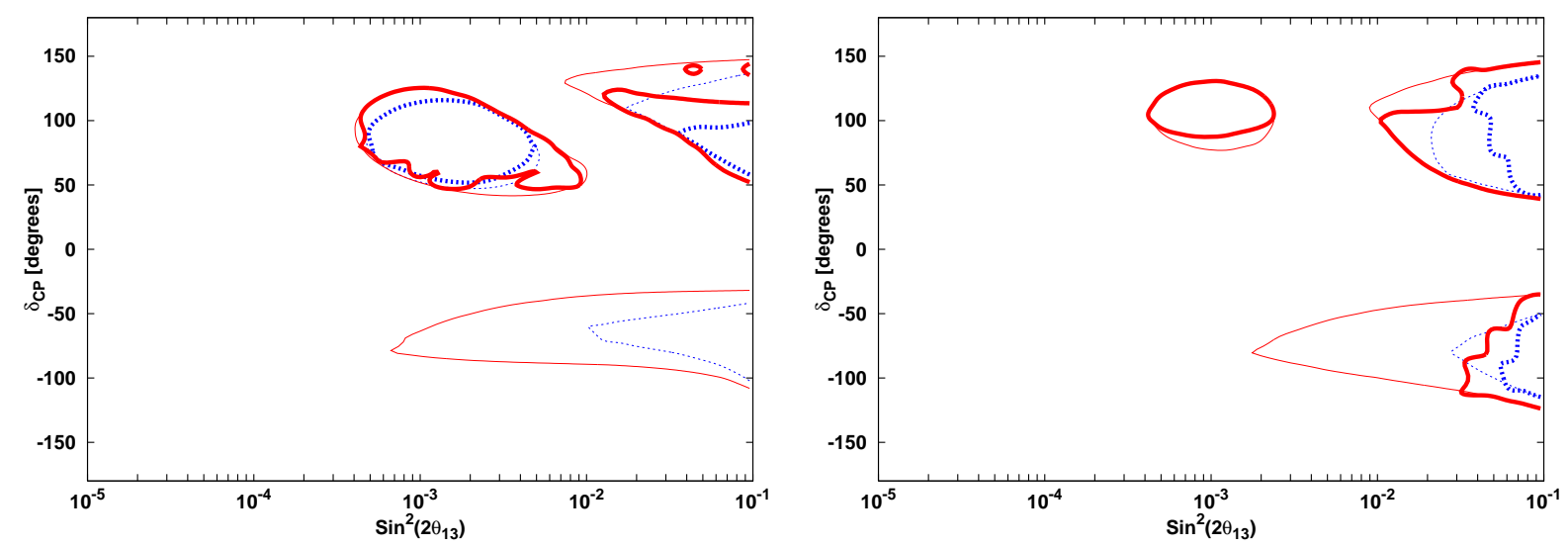

FIG. 7: CP-violation discovery potential at 99\% CL for setup III (left panel) and IV (right panel). In each case, we present the results for the beta-beam only (blue dotted lines) and the combination with the electron capture result (red solid lines), both without (thin lines) and with (thick lines) taking the hierarchy degeneracy into account.

cross section is poor. We could expect a similar sensitivity to CP-violation for a TASD or LAr detector with exposure a factor only a few times larger than the one considered in this analysis.

The effects of the hierarchy degeneracy are important, significantly more so for $\delta<0$, where there is a loss of sensitivity to CP-violation by a couple of orders of magnitude in $\sin ^{2} 2 \theta_{13}$. We can understand this effect by looking at Fig. 4, For the shorter CERN to Canfranc baseline, we note that for positive $\delta$ either the hierarchy can be determined or, where not, the hierarchy clone solution significantly overlaps with the true one. In the case of negative values of $\delta$, the hierarchy cannot be resolved even for large values of $\theta_{13}$; the clone solution stretches into $\mathrm{CP}$-conserving values preventing the possibility to determine CP-violation. The inclusion of the high energy EC channel helps in resolving the degeneracy, with CP-discovery down to $\sin ^{2} 2 \theta_{13} \sim 3 \times 10^{-5}$ at 99\% CL (left panel of Fig. 8).

The CERN to Boulby baseline (setups IV and IV-WC) has stronger degenerate effects, but it also provides a better ability to resolve them. The results with and without the hierarchy clone solution are not significantly different since, for the values for which one has sensitivity to CP-violation, the hierarchy can be resolved. For setup IV, the reach is limited to large values of $\sin ^{2} 2 \theta_{13}$. Using the WC detector, the much larger count rate brings significantly better results: CP-violation can be established for a large fraction of $\delta$ values, 

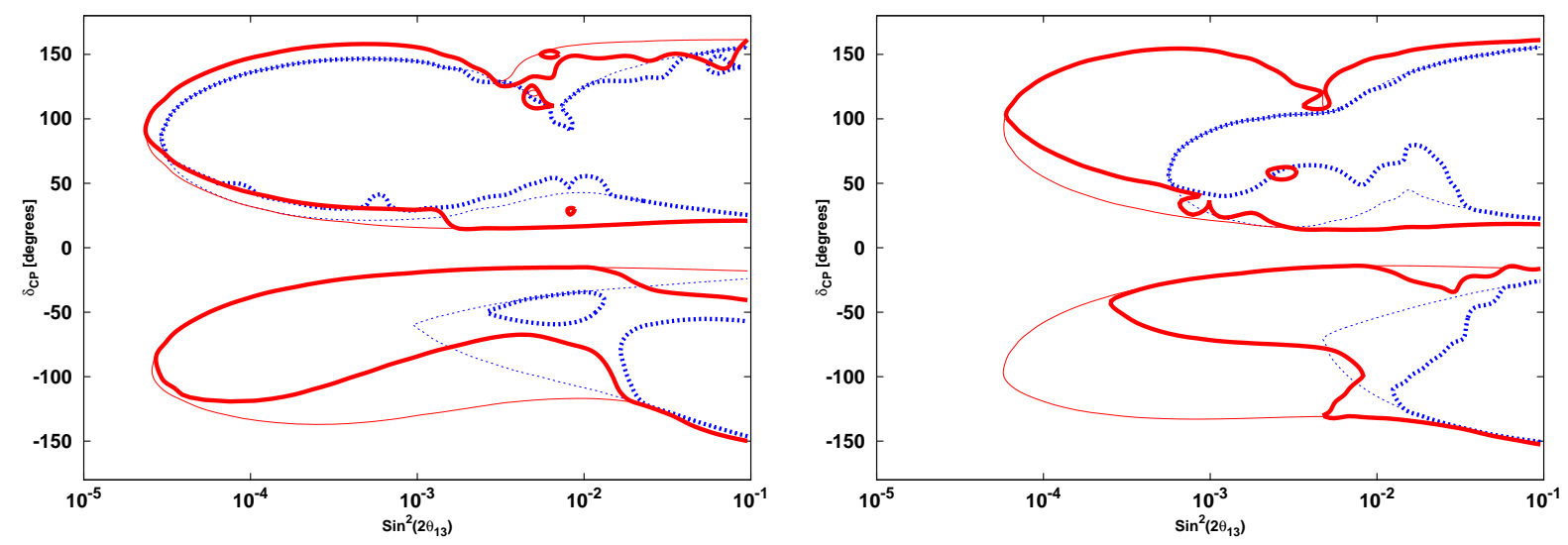

FIG. 8: Same as Fig. 7 but for setup III-WC (left panel) and setup IV-WC (right panel).

even for $\sin ^{2} 2 \theta_{13} \sim$ few $\times 10^{-4}$ at $99 \%$ CL.

Comparing the two locations of the detector, we notice that the shorter baseline (CERNCanfranc) has a slightly (significantly) better reach for CP-violation at positive (negative) values of $\delta$ than the longer baseline (CERN-Boulby). The longer option, however, performs slightly better at negative $\delta$ if the hierarchy is known to be normal and significantly better if the ordering is not determined. This is because the longer baseline can identify the neutrino mass hierarchy for these values of $\theta_{13}$, therefore resolving this degeneracy.

\section{Mass hierarchy determination}

In Fig. 9, we present the results for the neutrino mass hierarchy determination, but only for the setups with a 0.5 Mton WC detector (setups III-WC and IV-WC). We do not consider the CERN-Frejus cases; the shorter baseline being unable to distinguish the type of hierarchy.

In both cases, the contribution from the beta-beam channel is shown in blue dashed lines and the result for the combination with the electron capture channel is shown by the red solid lines. As matter effects are more important at high energies, we see that the inclusion of the electron capture flux improves the results, and in particular for the low values of $\sin ^{2} 2 \theta_{13}$ for which the measurement is possible. However, the chances to determine the mass hierarchy are very limited for the CERN-Canfranc baseline, never reaching more than $30 \%$ of the values of the $\mathrm{CP}$-violating phase $\delta$. On the other hand, the CERN-Boulby baseline, with its 

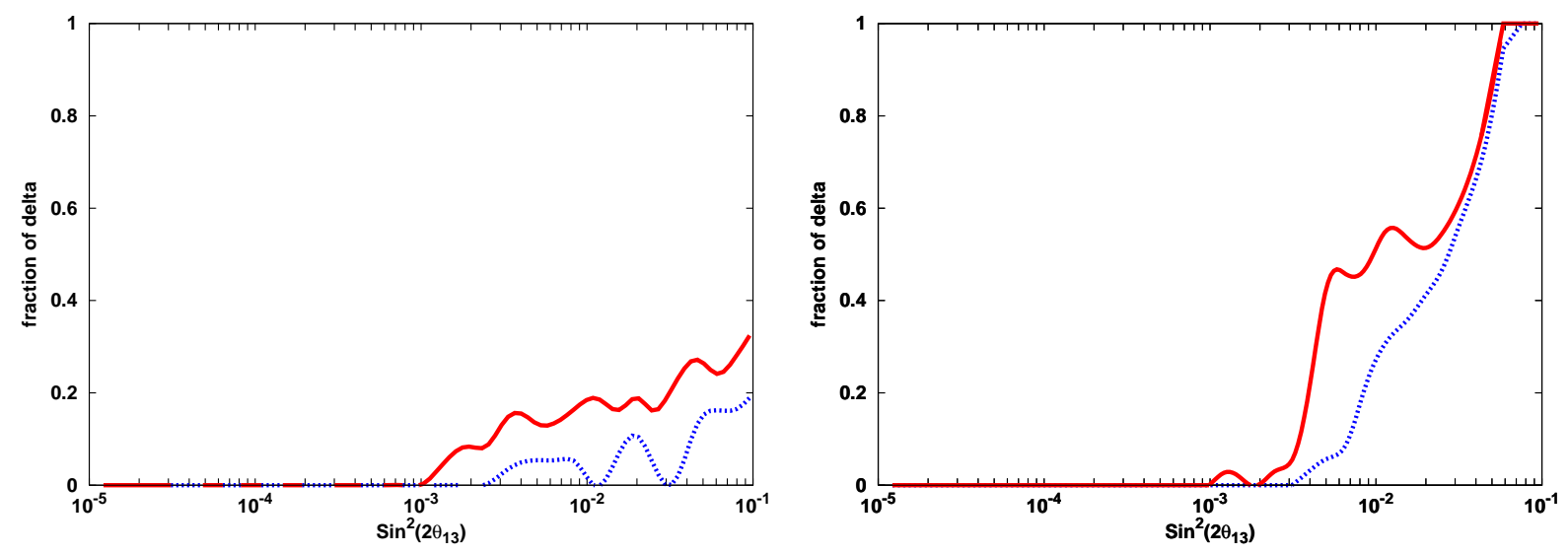

FIG. 9: Fraction of $\delta$ for which the neutrino mass hierarchy can be determined at 99\% CL for setup III-WC (left panel) and IV-WC (right panel). In each case, we present the results for the beta-beam only (blue dotted lines) and the combination with the electron capture result (red solid lines).

larger matter effect, represents a much more promising setup, for which the determination of the mass hierarchy would be possible for all values of $\delta$ for $\sin ^{2} 2 \theta_{13} \simeq$ few $\times 10^{-2}$, and with a $50 \%$ probability for $\sin ^{2} 2 \theta_{13} \simeq$ few $\times 10^{-3}$.

\section{DISCUSSION}

Let us note that by the time this experiment could possibly take place, there will be much better knowledge of the neutrino oscillation parameters, improving quite considerably the results presented here. Throughout this study, we have adopted a very conservative stance, namely the assumption that the errors on the neutrino parameters will remain the same by the time this experiment might start taking data. However, this is very likely not to be the case. Here, we present the results when no errors are included on the assumed values of the neutrino oscillation parameters, i.e., they are known with perfect precision. The actual performance of the experiment would lie in between these results and those presented in previous sections. For brevity, we only consider results for setups III-WC and IV-WC.

The CP-discovery potential is shown in Fig. 10, where the left (right) panel represents the setup III-WC (setup IV-WC) and the beta-beam only (beta-beam plus electron capture) performance is shown by the dashed blue lines (red solid lines). From Figs. 8 and 10, the 

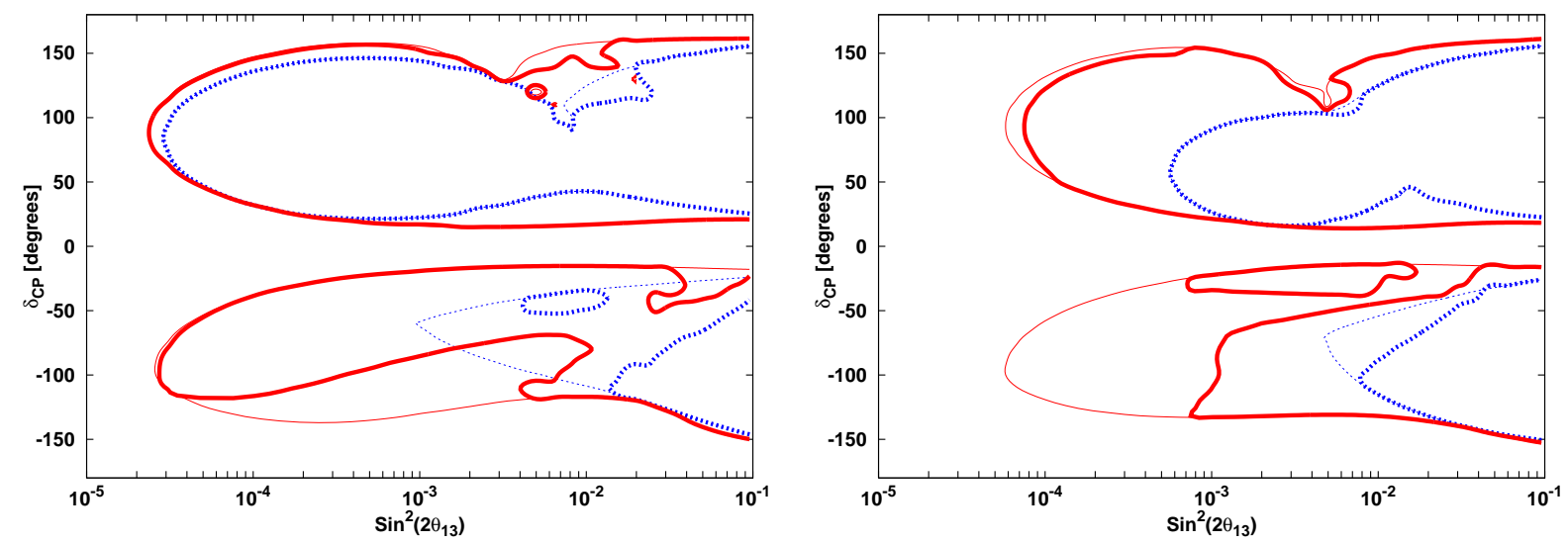

FIG. 10: Same as Fig. 8, but with negligible error in the value of the assumed neutrino parameters.

difference between use of the present uncertainty in the neutrino oscillation parameters and no uncertainty is seen to be small.

On the other hand, we show in Fig. 11 the extent to which mass hierarchy determination could improve with better knowledge of the assumed neutrino oscillation parameters. Again, we only show the case of setup III-WC (left panel) and setup IV-WC (right panel) with the same designations as previous, i.e., blue dashed lines for the beta-beam only contribution and red solid lines for the performance of the total flux (i.e., adding the electron capture flux). The difference between Figs. 9 and 11 is substantial. While the qualitative behaviour of the relative contribution of the beta-beam only part is very similar, in the case of perfect knowledge of the assumed parameters, the determination of the neutrino mass hierarchy is possible for $50 \%$ of the values of $\delta$ down to $\sin ^{2} 2 \theta_{13} \simeq 8 \times 10^{-4}$ for setup IV-WC (right panel of Fig. 11). This improvement represents about an order of magnitude with respect to the case depicted in the right panel of Fig. 9.

\section{SUMMARY AND CONCLUSIONS}

Determining the value of $\theta_{13}$, the type of neutrino mass ordering and the presence of leptonic CP-violation will be one of the main goals of the future experimental neutrino program which is under intensive discussion at present. In the present article we have studied a new type of experimental setup which combines a beta-beam with an electron capture beam. This can be achieved naturally by using nuclei which can decay into both 

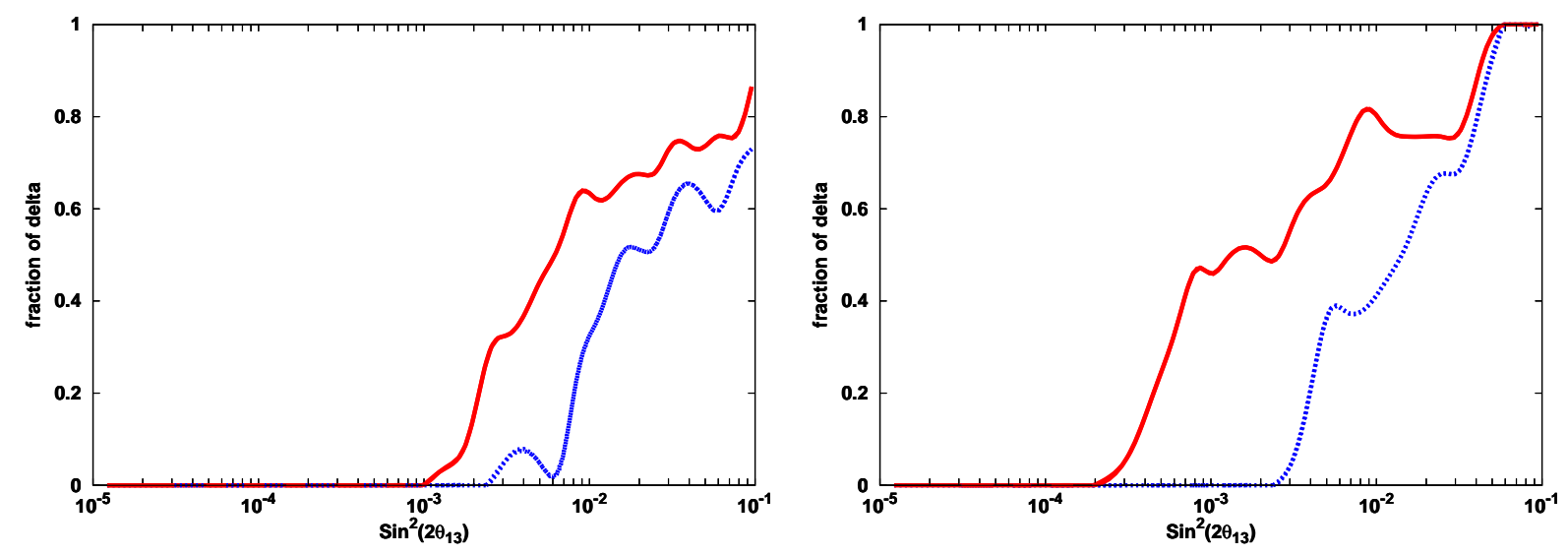

FIG. 11: Same as Fig. 9, but with negligible error in the value of the assumed neutrino parameters.

channels. We have studied this idea using the nuclide ${ }^{156} \mathrm{Yb}$ which has favourable betadecay and electron capture branching ratios, and only a small alpha decay contribution. This combination is very powerful as the EC channel provides a high energy signal at a well known energy, while the beta-beam provides coverage of the first and second oscillation maxima. The allowed regions in the $\left(\theta_{13}, \delta\right)$ plane for the two separate channels have a limited overlap resulting in a good resolution of the intrinsic degeneracy. We have understood the main features of this synergy by an analytical study of the oscillation probability. It should be stressed that this setup does not require two polarities but reaches a very good sensitivity by only using the neutrino channel through full exploitation of the oscillatory pattern of the appearance probability.

We have performed a detailed study of the dependence of the physics reach of this experimental technique by considering six different setups: two values for the ion boost factor $\gamma=166$ and 369; two choices for the detector: a 50 kton TASD or LiAr and a 0.5 Mton WC detector; and three baselines: CERN-Frejus, CERN-Canfranc, CERN-Boulby. This allowed us to study the impact of the count rate, choice of baseline and the tuning of the energy of the beta-beam and EC beam to the oscillatory pattern. We find that the setups with low gamma and 50 kton detectors have very poor physics reach, owing to the limited event rates. The information on CP-violation is mainly provided by the high energy EC signal. We studied the options with $\gamma=369$, the highest value of the boost factor allowed by an upgraded SPS. Setups III and III-WC, which use the CERN-Canfranc baseline, have larger count rates and a better tuning of the beam to the oscillatory pattern, with respect 
to their CERN-Boulby counterparts: setups IV and IV-WC. This results in a very good ability to measure the parameters, see Fig. 8. In particular these setups provide the best sensitivity to $\mathrm{CP}$-violation for positive values of $\delta$. However, for negative $\delta$, owing to the relatively short distance, the type of hierarchy can be resolved only for very large values of $\theta_{13}$. The sign-degeneracy prevents discovery of CP-violation in this case, see Fig. 4. The CERN-Boulby setups, IV and IV-WC, suffer from smaller count rates and poor tuning of the beta-beam to the oscillation pattern. However, they provide a much better determination of the hierarchy and possess a good reach to CP-violation for $\delta<0$, even if the mass

ordering is not known. Comparing the two baseline options, if the hierarchy is known to be normal from other neutrino experiments, the CERN-Canfranc option has an improved physics reach, while if the ordering is not known, the CERN-Boulby baseline outperforms the shorter option. For the high statistics scenario, one gets sensitivity to CP-violation down to values of $\sin ^{2} 2 \theta_{13} \sim 3 \times 10^{-5}$ at $99 \%$ CL for a WC detector at Canfranc, and $\sin ^{2} 2 \theta_{13} \sim 10^{-4}$ for a WC detector at Boulby.

In conclusion, we have presented the novel idea of using a single beam which combines neutrinos from beta and electron capture decays and have demonstrated the physics reach of several possible setups. We have shown that the combination of these two types of beams achieves remarkable results. This could naturally be done with the use ${ }^{156} \mathrm{Yb}$, which has comparable beta-beam and electron capture branching ratios. As both beams are produced from a single isotope, this combination cannot be further optimised. An analogous setup would be obtained if a beta-beam and an electron capture beam sourced from different ions are combined. In this case, further optimisation of the experiment would be allowed, for suitable choices of baselines, Lorentz boost factors, detector size and technology, possibly achieving an even better physics reach than the one found in the present study.

\section{Acknowledgments}

We would like to thank O. Mena and W. Winter for useful discussions. CE acknowledges the support from the Spanish Generalitat Valenciana and thanks the IPPP for hospitality at the initial stage of this study. CO acknowledges the support of a STFC studentship and overseas fieldwork support. In addition, CO and SPR would like to thank the Department de Fisica Teòrica, Universitat de València for hospitality during the middle stages of this work. 
SPR is supported by the Portuguese FCT through the projects POCI/FP/81919/2007, CERN/FP/83503/2008 and CFTP-FCT UNIT 777, which are partially funded through POCTI (FEDER). SPR is also partially supported by the Spanish Grant FPA2005-01678 of the MCT. CO and SP acknowledge the support of CARE, contract number RII3-CT-2003506395.

[1] Y. Ashie et al. [Super-Kamiokande Collaboration], Phys. Rev. D 71 (2005) 112005 [arXiv:hep-ex/0501064]; J. Hosaka et al. [Super-Kamiokande Collaboration], neutrinos in Super-Kamiokande," Phys. Rev. D 74 (2006) 032002 arXiv:hep-ex/0604011]; H. Sekiya for the Super-Kamiokande Collaboration, arXiv:0810.0595 [astro-ph].

[2] M. Ambrosio et al. [MACRO Collaboration], Eur. Phys. J. C 36 (2004) 323; M. C. Sanchez et al. [Soudan 2 Collaboration], Phys. Rev. D 68, 113004 (2003) arXiv:hep-ex/0307069].

[3] Y. Fukuda et al. [Kamiokande Collaboration], Phys. Rev. Lett. 77 (1996) 1683; B. T. Cleveland et al., Astrophys. J. 496 (1998) 505; W. Hampel et al. [GALLEX Collaboration], Phys. Lett. B 447 (1999) 127; J. N. Abdurashitov et al. [SAGE Collaboration], J. Exp. Theor. Phys. 95 (2002) 181 [Zh. Eksp. Teor. Fiz. 122 (2002) 211] [arXiv:astro-ph/0204245]; T. A. Kirsten [GNO Collaboration], Nucl. Phys. Proc. Suppl. 118 (2003) 33.

[4] S. Fukuda et al. [Super-Kamiokande Collaboration], Phys. Lett. B 539 (2002) 179 arXiv:hep-ex/0205075]. J. P. Cravens et al. [Super-Kamiokande Collaboration], Phys. Rev. D 78 (2008) 032002 arXiv:0803.4312 [hep-ex]];

[5] Q. R. Ahmad et al. [SNO Collaboration], Phys. Rev. Lett. 87 (2001) 071301 arXiv:nucl-ex/0106015]; ibid. 89 (2002) 011301 arXiv:nucl-ex/0204008]; and ibid. 89 (2002) 011302 arXiv:nucl-ex/0204009]. S. N. Ahmed et al. [SNO Collaboration], Phys. Rev. Lett. 92 (2004) 181301 [arXiv:nucl-ex/0309004]; B. Aharmim et al. [SNO Collaboration], Phys. Rev. C 72 (2005) 055502 arXiv:nucl-ex/0502021.

[6] M. Apollonio et al. [CHOOZ Collaboration], Phys. Lett. B 466 (1999) 415 arXiv:hep-ex/9907037; M. Apollonio et al. [CHOOZ Collaboration], Eur. Phys. J. C 27 (2003) 331 arXiv:hep-ex/0301017.

[7] F. Boehm et al., Phys. Rev. Lett. 84 (2000) 3764 arXiv:hep-ex/9912050]; Phys. Rev. D 62 (2000) 072002 arXiv:hep-ex/0003022; and ibid. 64 (2001) 112001 arXiv:hep-ex/0107009. 
[8] K. Eguchi et al. [KamLAND Collaboration], Phys. Rev. Lett. 90 (2003) 021802 arXiv:hep-ex/0212021]; T. Araki et al. [KamLAND Collaboration], Phys. Rev. Lett. 94 (2005) 081801 arXiv:hep-ex/0406035. S. Abeet al. [KamLAND Collaboration], Phys. Rev. Lett. 100 (2008) 221803 arXiv:0801.4589 [hep-ex]].

[9] M. H. Ahn et al. [K2K Collaboration], Phys. Rev. D 74 (2006) 072003 arXiv:hep-ex/0606032].

[10] D. G. Michael et al. [MINOS Collaboration], Phys. Rev. Lett. 97 (2006) 191801 arXiv:hep-ex/0607088; P. Adamsonet al. [MINOS Collaboration], arXiv:0806.2237 [hep-ex]; arXiv:0807.2424 [hep-ex].

[11] T. Schwetz, M. Tortola and J. W. F. Valle, New J. Phys. 10 (2008) 113011 arXiv:0808.2016 [hep-ph]].

[12] G. L. Fogli, E. Lisi, A. Marrone, A. Palazzo and A. M. Rotunno, Phys. Rev. Lett. 101, 141801 (2008) arXiv:0806.2649 [hep-ph]].

[13] H. L. Ge, C. Giunti and Q. Y. Liu, arXiv:0810.5443 [hep-ph].

[14] A. Bandyopadhyay et al. [ISS Physics Working Group], arXiv:0710.4947 [hep-ph].

[15] "Euro- $\nu$ : High Intensity Neutrino Oscillation Facility in Europe", FP7-infrastructures-2007-1 project number 212372 .

[16] F. Ardellier et al. [Double Chooz Collaboration], arXiv:hep-ex/0606025. X. Guo et al. [Daya Bay Collaboration] hep-ex/0701029; S. B. Kim [RENO Collaboration], AIP Conf. Proc. 981 (2008) 205 [J. Phys. Conf. Ser. 120 (2008) 052025]; H. Nunokawa [Angra Neutrino Collaboration], AIP Conf. Proc. 981 (2008) 208.

[17] Y. Hayato et al., Letter of Intent, available at http://neutrino.kek.jp/jhfnu/.

[18] D. S. Ayres et al. [NOvA Collaboration], hep-ex/0503053. FERMILABPROPOSAL-0929, March 21, 2005. Revised nova Proposal available at http://www-nova.fnal.gov/NOvA_Proposal/Revised_NOvA_Proposal.html.

[19] V. Barger, P. Huber, D. Marfatia and W. Winter, Phys. Rev. D 76 (2007) 053005 arXiv:hep-ph/0703029]; V. Barger et al., arXiv:0705.4396 [hep-ph].

[20] S. Geer, Phys. Rev. D $5 \mathbf{5 7}$ (1998) 6989 [Erratum-ibid. D 59 (1999) 039903] arXiv:hep-ph/9712290 A. De Rújula, M. B. Gavela and P. Hernández, Nucl. Phys. B 547 (1999) 21 arXiv:hep-ph/9811390]; V. Barger, S. Geer, R. Raja and K. Whisnant, Phys. Rev. D 62 (2000) 013004 arXiv:hep-ph/9911524]; M. Freund, M. Lindner, S. T. Petcov and A. Romanino, Nucl. Phys. B 578 (2000) 27 arXiv:hep-ph/9912457. 
[21] S. Geer, O. Mena and S. Pascoli, Phys. Rev. D 75, 093001 (2007) arXiv:hep-ph/0701258; A. D. Bross, M. Ellis, S. Geer, O. Mena and S. Pascoli, Phys. Rev. D 77, 093012 (2008) arXiv:0709.3889 [hep-ph]].

[22] P. Zucchelli, Phys. Lett. B 532 (2002) 166.

[23] M. Mezzetto, J. Phys. G 29 (2003) 1781 arXiv:hep-ex/0302005]; and ibid. 29 (2003) 1771.

[24] J. Burguet-Castell, M. B. Gavela, J. J. Gomez-Cadenas, P. Hernandez and O. Mena, Nucl. Phys. B 608 (2001) 301 arXiv:hep-ph/0103258.

[25] H. Minakata and H. Nunokawa, JHEP 0110 (2001) 001 arXiv:hep-ph/0108085.

[26] G. L. Fogli and E. Lisi, Phys. Rev. D 54 (1996) 3667 [arXiv:hep-ph/9604415].

[27] V. Barger, D. Marfatia and K. Whisnant, arXiv:hep-ph/0112119 Phys. Rev. D 65 (2002) 073023 arXiv:hep-ph/0112119].

[28] M. Freund, P. Huber and M. Lindner, Nucl. Phys. B 615 (2001) 331 arXiv:hep-ph/0105071. T. Kajita, H. Minakata and H. Nunokawa, Phys. Lett. B 528 (2002) 245 arXiv:hep-ph/0112345]; H. Minakata, H. Nunokawa and S. J. Parke, Phys. Rev. D 66 (2002) 093012 arXiv:hep-ph/0208163] ; P. Huber, M. Lindner and W. Winter, Nucl. Phys. B 645 (2002) 3 arXiv:hep-ph/0204352; A. Donini, D. Meloni and S. Rigolin, JHEP 0406 (2004) 011 arXiv:hep-ph/0312072; M. Aoki, K. Hagiwara and N. Okamura, Phys. Lett. B 606 (2005) 371 arXiv:hep-ph/0311324; O. Yasuda, New J. Phys. 6 (2004) 83 arXiv:hep-ph/0405005; O. Mena and S. J. Parke, Phys. Rev. D 72 (2005) 053003 arXiv:hep-ph/0505202.

[29] D. Meloni, O. Mena, C. Orme, S. Palomares-Ruiz and S. Pascoli, JHEP 0807 (2008) 115 arXiv:0802.0255 [hep-ph]].

[30] S. T. Petcov and M. Piai, Phys. Lett. B 533 (2002) 94 arXiv:hep-ph/0112074]; S. Choubey, S. T. Petcov and M. Piai, Phys. Rev. D 68 (2003) 113006 arXiv:hep-ph/0306017; J. Learned, S. T. Dye, S. Pakvasa and R. C. Svoboda, Phys. Rev. D 78 (2008) 071302 arXiv:hep-ex/0612022; L. Zhan, Y. Wang, J. Cao and L. Wen, Phys. Rev. D 78 (2008) 111103 arXiv:0807.3203 [hep-ex]]; and arXiv:0901.2976 [hep-ex].

[31] Y. F. Wang, K. Whisnant, Z. h. Xiong, J. M. Yang and B. L. Young [VLBL Study Group H2B-4], Phys. Rev. D 65 (2002) 073021 arXiv:hep-ph/0111317); J. Burguet-Castell, M. B. Gavela, J. J. Gomez-Cadenas, P. Hernandez and O. Mena, Nucl. Phys. B 646 (2002) 301 arXiv:hep-ph/0207080]; K. Whisnant, J. M. Yang and B. L. Young, Phys. Rev. D 67 (2003) 013004 arXiv:hep-ph/0208193; V. Barger, D. Marfatia and K. Whisnant, Phys. 
Lett. B 560 (2003) 75 arXiv:hep-ph/0210428]; P. Huber, M. Lindner and W. Winter, Nucl. Phys. B 654 (2003) 3 arXiv:hep-ph/0211300; H. Minakata, H. Nunokawa and S. J. Parke, Phys. Rev. D 68 (2003) 013010 arXiv:hep-ph/0301210; A. Donini, Nucl. Phys. B 710 (2005) 402 arXiv:hep-ph/0406132; O. Mena and S. J. Parke, Phys. Rev. D 70 (2004) 093011 arXiv:hep-ph/0408070]; A. Donini, E. Fernández-Martínez and S. Rigolin, Phys. Lett. B 621 (2005) 276 arXiv:hep-ph/0411402]; P. Huber, M. Maltoni and T. Schwetz, Phys. Rev. D 71 (2005) 053006 arXiv:hep-ph/0501037]; O. Mena, Mod. Phys. Lett. A 20 (2005) 1 [arXiv:hep-ph/0503097]; A. Blondel et al., Acta Phys. Polon. B 37 (2006) 2077 arXiv:hep-ph/0606111; ; P. Huber, M. Lindner, M. Rolinec and W. Winter, Phys. Rev. D 74 (2006) 073003 arXiv:hep-ph/0606119]; O. Mena, H. Nunokawa and S. J. Parke, Phys. Rev. D 75 (2007) 033002 arXiv:hep-ph/0609011]. O. Mena, hep-ph/0609031. A. Jansson, O. Mena, S. J. Parke and N. Saoulidou, Phys. Rev. D 78 (2008) 053002 arXiv:0711.1075 [hep-ph]].

[32] P. Huber, M. Lindner, T. Schwetz and W. Winter, Nucl. Phys. B 665 (2003) 487 arXiv:hep-ph/0303232; P. Huber, M. Lindner, M. Rolinec, T. Schwetz and W. Winter, Phys. Rev. D 70 (2004) 073014 arXiv:hep-ph/0403068.

[33] H. Minakata and H. Nunokawa, Phys. Lett. B 413 (1997) 369 arXiv:hep-ph/9706281.

[34] A. Donini, D. Meloni and P. Migliozzi, Nucl. Phys. B 646 (2002) 321 arXiv:hep-ph/0206034; D. Autiero et al., Eur. Phys. J. C 33 (2004) 243 arXiv:hep-ph/0305185.

[35] V. Barger, D. Marfatia and K. Whisnant, Phys. Rev. D 66 (2002) 053007 arXiv:hep-ph/0206038.

[36] O. Mena Requejo, S. Palomares-Ruiz and S. Pascoli, Phys. Rev. D 72 (2005) 053002 arXiv:hep-ph/0504015; and ibid. 73 (2006) 073007 arXiv:hep-ph/0510182.

[37] M. Ishitsuka, T. Kajita, H. Minakata and H. Nunokawa, Phys. Rev. D 72 (2005) 033003 arXiv:hep-ph/0504026]; K. Hagiwara, N. Okamura and K. i. Senda, Phys. Lett. B 637 (2006) 266 arXiv:hep-ph/0504061. T. Kajita, H. Minakata, S. Nakayama and H. Nunokawa, Phys. Rev. D 75 (2007) 013006 arXiv:hep-ph/0609286]; K. Hagiwara and N. Okamura, arXiv:0901.1517 [hep-ph].

[38] P. Coloma, A. Donini, E. Fernandez-Martinez and J. Lopez-Pavon, JHEP 0805 (2008) 050 arXiv:0712.0796 [hep-ph]].

[39] S. T. Petcov, Phys. Lett. B 434 (1998) $321 \quad$ [Erratum-ibid. $\quad$ B 444 (1998) 584] arXiv:hep-ph/9805262; E. K. Akhmedov, Nucl. Phys. B 538 (1999) 25 
arXiv:hep-ph/9805272; E. K. Akhmedov, A. Dighe, P. Lipari and A. Y. Smirnov, Nucl. Phys. B 542 (1999) 3 arXiv:hep-ph/9808270; M. V. Chizhov, M. Maris and S. T. Petcov, hep-ph/9810501; M. V. Chizhov and S.T. Petcov, Phys. Rev. Lett. 83 (1999) 1096 arXiv:hep-ph/9903399; ibid. 85, 3979 (2000); and Phys. Rev. D 63 (2001) 073003 arXiv:hep-ph/9903424]; E. K. Akhmedov, M. Maltoni and A. Y. Smirnov, Phys. Rev. Lett. 95 (2005) 211801 arXiv:hep-ph/0506064 and JHEP 0705 (2007) 077 arXiv:hep-ph/0612285.

[40] M. C. Bañuls, G. Barenboim and J. Bernabéu, Phys. Lett. B 513 (2001) 391 arXiv:hep-ph/0102184; J. Bernabéu and S. Palomares-Ruiz, hep-ph/0112002; and Nucl. Phys. Proc. Suppl. 110 (2002) 339 [arXiv:hep-ph/0201090].

[41] J. Bernabéu, S. Palomares-Ruiz, A. Pérez and S. T. Petcov, Phys. Lett. B 531 (2002) 90 arXiv:hep-ph/0110071]; S. Palomares-Ruiz and J. Bernabéu, Nucl. Phys. Proc. Suppl. 138 (2005) 398 arXiv:hep-ph/0312038.

[42] J. Bernabéu, S. Palomares Ruiz and S. T. Petcov, Nucl. Phys. B 669 (2003) 255 arXiv:hep-ph/0305152]; S. Palomares-Ruiz and S. T. Petcov, Nucl. Phys. B 712 (2005) 392 arXiv:hep-ph/0406096; S. T. Petcov and S. Palomares-Ruiz, hep-ph/0406106; S. T. Petcov and T. Schwetz, Nucl. Phys. B 740 (2006) 1 arXiv:hep-ph/0511277.

[43] D. Indumathi and M. V. N. Murthy, Phys. Rev. D 71 (2005) 013001 arXiv:hep-ph/0407336; R. Gandhi, P. Ghoshal, S. Goswami, P. Mehta and S. Uma Sankar, Phys. Rev. D 73 (2006) 053001 arXiv:hep-ph/0411252; and arXiv:hep-ph/0506145. H. Back et al., arXiv:hep-ex/0412016; D. Indumathi, M. V. N. Murthy, G. Rajasekaran and N. Sinha, Phys. Rev. D 74 (2006) 053004 arXiv:hep-ph/0603264]; R. Gandhi et al., Phys. Rev. D 76 (2007) 073012 [arXiv:0707.1723 [hep-ph]]; O. Mena, I. Mocioiu and S. Razzaque, Phys. Rev. D 78 (2008) 093003 [arXiv:0803.3044 [hep-ph]]; R. Gandhi, P. Ghoshal, S. Goswami and S. Uma Sankar, Phys. Rev. D 78 (2008) 073001 [arXiv:0807.2759 [hep-ph]].

[44] C. Lunardini and A. Y. Smirnov, Nucl. Phys. B 616 (2001) 307 arXiv:hep-ph/0106149]; and JCAP 0306 (2003) 009 arXiv:hep-ph/0302033; K. Takahashi and K. Sato, Phys. Rev. D 66 (2002) 033006 arXiv:hep-ph/0110105; and Prog. Theor. Phys. 109 (2003) 919 arXiv:hep-ph/0205070; A. S. Dighe, M. T. Keil and G. G. Raffelt, JCAP 0306 (2003) 005 arXiv:hep-ph/0303210; and ibid. 0306 (2003) 006 arXiv:hep-ph/0304150; A. S. Dighe, M. Kachelriess, G. G. Raffelt and R. Tomas, JCAP 0401 (2004) 004 arXiv:hep-ph/0311172; A. Bandyopadhyay, S. Choubey, S. Goswami and K. Kar, hep-ph/0312315; V. Barger, P. Hu- 
ber and D. Marfatia, Phys. Lett. B 617 (2005) 167 arXiv:hep-ph/0501184].

[45] B. Dasgupta, A. Dighe and A. Mirizzi, Phys. Rev. Lett. 101 (2008) 171801 arXiv:0802.1481 [hep-ph]].

[46] P. I. Krastev and S. T. Petcov, Phys. Lett. B 205 (1988) 84; J. Arafune and J. Sato, Phys. Rev. D 55 (1997) 1653 [arXiv:hep-ph/9607437]; J. Bernabéu, Proc. WIN'99, World Scientific (2000), p. 227, hep-ph/9904474; M. Freund, M. Lindner and A. Romanino, Nucl. Phys. B 562 (1999) 29 arXiv:hep-ph/9903308.

[47] J. Bernabéu and M. C. Bañuls, Nucl. Phys. Proc. Suppl. 87 (2000) 315 arXiv:hep-ph/0003299.

[48] J. Burguet-Castell, D. Casper, J. J. Gomez-Cadenas, P. Hernandez and F. Sanchez, Nucl. Phys. B 695 (2004) 217 arXiv:hep-ph/0312068]; J. Burguet-Castell, D. Casper, E. Couce, J. J. Gomez-Cadenas and P. Hernandez, Nucl. Phys. B 725 (2005) 306 arXiv:hep-ph/0503021.

[49] P. Huber, M. Lindner, M. Rolinec and W. Winter, Phys. Rev. D 73 (2006) 053002 arXiv:hep-ph/0506237.

[50] A. Donini and E. Fernandez-Martinez, Phys. Lett. B 641 (2006) 432 arXiv:hep-ph/0603261.

[51] A. Donini et al., arXiv:hep-ph/0511134; and Eur. Phys. J. C 48 (2006) 787 arXiv:hep-ph/0604229; A. Donini et al., Eur. Phys. J. $\quad$ C 53 (2008) 599 arXiv:hep-ph/0703209.

[52] S. K. Agarwalla, A. Raychaudhuri and A. Samanta, Phys. Lett. B 629 (2005) 33 arXiv:hep-ph/0505015]; S. K. Agarwalla, S. Choubey and A. Raychaudhuri, Nucl. Phys. B 771 (2007) 1 arXiv:hep-ph/0610333; ibid. 798 (2008) 124 arXiv:0711.1459 [hep-ph]]; and ibid. 805 (2008) 305 [arXiv:0804.3007 [hep-ph]]; S. K. Agarwalla, S. Choubey, S. Goswami and A. Raychaudhuri, Phys. Rev. D 75 (2007) 097302 arXiv:hep-ph/0611233]; S. K. Agarwalla, S. Choubey, A. Raychaudhuri and W. Winter, JHEP 0806 (2008) 090 arXiv:0802.3621 [hep-ex]].

[53] J. Bernabeu, J. Burguet-Castell, C. Espinoza and M. Lindroos, JHEP 0512 (2005) 014 arXiv:hep-ph/0505054; Nucl. Phys. Proc. Suppl. 155 (2006) 222 arXiv:hep-ph/0510278; PoS HEP2005 (2006) 182 arXiv:hep-ph/0512297]; arXiv:hep-ph/0512299; PoS HEP2005 (2006) 365; J. Bernabeu and C. Espinoza, arXiv:hep-ph/0605132; C. Espinoza, J. Bernabeu, J. Burguet-Castell and M. Lindroos, AIP Conf. Proc. 885 (2007) 89. 
[54] J. Bernabeu and C. Espinoza, Phys. Lett. B 664 (2008) 285 arXiv:0712.1034 [hep-ph]]; arXiv:hep-ph/0612316; AIP Conf. Proc. 917 (2007) 24; J. Phys. Conf. Ser. 110 (2008) 082006 arXiv:0710.5615 [hep-ph]].

[55] J. Sato, Phys. Rev. Lett. 95 (2005) 131804 arXiv:hep-ph/0503144].

[56] M. Rolinec and J. Sato, JHEP 0708 (2007) 079 arXiv:hep-ph/0612148.

[57] B. Rubio and W. Gelletly, Lect. Notes Phys. 764 (2009) 99.

[58] M. R. Bhat, Nucl. Data Sheets 89 (2000) 797.

[59] C. W. Reich, Nucl. Data Sheets 99 (2003) 753.

[60] A. Algora et al., Proc. XXXII Symposium on Nuclear Physics, Cocoyoc, Morelos, Mexico (2009); M. E. Estévez et al., DPG Spring Meeting, Bochum, Germany (2009); A. Algora, E. Estévez and B. Rubio, private communication.

[61] H. Keller et al., Z. Phys. A 352 (1995) 1.

[62] E. Nácher et al., GSI Sci. Report 2001, 8.

[63] M. Lindroos, private communication.

[64] A. Cervera et al., Nucl. Phys. B 579 (2000) 17 [Erratum-ibid. B 593 (2001) 731] arXiv:hep-ph/0002108.

[65] E. K. Akhmedov, R. Johansson, M. Lindner, T. Ohlsson and T. Schwetz, JHEP 0404 (2004) 078 arXiv:hep-ph/0402175]. 